$\begin{array}{ll}\text { Italique } & \text { Italique } \\ \text { Poésie italienne de la Renaissance }\end{array}$

XVI | 2013

Varia

\title{
I capitoli di Francesco Maria Molza. Storia esterna e restauri testuali
}

\section{Franco Pignatti}

\section{(2) OpenEdition}

\section{Journals}

\section{Edizione digitale}

URL: http://journals.openedition.org/italique/367

DOI: $10.4000 /$ italique.367

ISSN: 1663-4438

\section{Editore}

Librairie Droz

\section{Edizione cartacea}

Data di pubblicazione: 1 dicembre 2013

Paginazione: 11-67

ISBN: 978-2-600-01779-4

ISSN: 1423-3983

\section{Notizia bibliografica digitale}

Franco Pignatti, «I capitoli di Francesco Maria Molza. Storia esterna e restauri testuali », Italique [Online], XVI | 2013, online dal 01 décembre 2016, consultato il 01 mai 2019. URL : http:// journals.openedition.org/italique/367 ; DOI : 10.4000/italique.367 


$$
\text { FrANCO PIGNATTI }
$$

$$
\text { I C A P I T O L I }
$$

D I FRA NCESCO MARIA MOLZA.

$$
\text { STORIA ESTERNA }
$$

E RESTAURI TESTUALI 



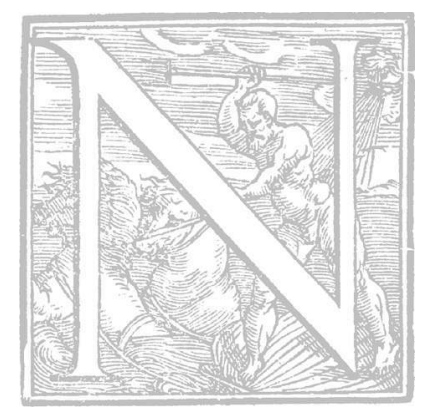

ella prima metà degli anni Trenta Molza fu in contatto con $i$ letterati che si riunivano a Roma intorno al gentiluomo mantovano Uberto Strozzi ed è consuetudine indicare, sulla scorta di un passo della Libraria e di uno dei Mondi di Anton Francesco Doni, ${ }^{\mathrm{I}}$ con il nome di Accademia della Vigna o dei Vignaiuoli. Doni riferisce dell'uso di questi letterati di assumere pseudonimi richiamanti vegetali orticoli o utensili necessari alla loro coltivazione, nonché degli argomenti attinenti all'agricoltura trattati nelle loro opere. I luoghi doniani sono però muti quanto allidentità del patrono e dei membri della conventicola, nonché alla sua cronologia. Il nome dello Strozzi, e di un buon numero di partecipanti alle riunioni letterarie che si tenevano nella sua dimora romana, si ricava dalla lettera di dedica dell'edizione postuma delle Institutioni al comporre in ogni sorte di rima della lingua volgare di Mario Equicola (Milano, F.M. Calvo, I54I), a firma del non altrimenti noto Marco Sabino e diretta appunto allo Strozzi. Tra «tutti i piu famosi Academici che fossero in Corte» di Roma e frequentavano il diporto strozziano Sabino distingue un drappello specialmente dedito ad argomenti piacevoli e burleschi, senza però indicare un epiteto che distinguesse questi verseggiatori tra $i$ letterati che animavano il cenacolo, né richiamare la consuetudine di attribuirsi nomi georgici, secondo quanto riportato da Doni.

La notorietà delle testimonianze citate, sotto gli occhi di tutti da quando Francesco Saverio Quadrio le utilizzò per la scheda sull'Accademia nel Della storia e della ragione d'ogni poesia e Girolamo Tiraboschi incluse la lettera di Sabino nell' ampio capitolo dedicato all'evoluzione dell'Accademia Romana e ad «altre accademie a' tempi di Paolo III» della sua Storia della letteratura italiana, ${ }^{2}$ ci dispensa dal riportarle per esteso, ma necessita qualche corollario per valutare bene il loro peso al fine di certificare o no la presenza di Molza nell'accolita vignainola.

La lettera di Sabino, generosa nel rammentare al destinatario i frequentatori del suo ridotto, quando esso non esisteva più da diverso tempo, tace il nome del letterato modenese. Nel gruppo di coloro che coltivavano la musa burlesca Sabino si concede invece qualche calibrato gindizio: 
Francesco Berni si distingue per le sue «argute facezie», di Giovanni Mauro d'Arcano spiccano le «attrattive piacevolezze», «ingegnosi concetti» sono accreditati a Giovanni Della Casa, «ameno» è giudicato il lucchese Giuseppe Giova; seguono, sprovvisti di esornativi, Lelio Capilupi, Agnolo Firenzuola, Giovan Francesco Bini «con molti altri》. Quando Sabino esprimeva questi giudizi se n'erano andati $i$ primi due personaggi posti in cima alla lista: Berni era morto il 26 maggio I535, nell'agosto dell'anno dopo era perito in un incidente di caccia Arcano, giudicato tra i contemporanei all'altezza del padre eponimo di questo genere di poesia, al punto di essergli accostato e addirittura anteposto nei frontespizi delle prime collettanee a stampa. ${ }^{3}$ Scomparsi $i$ dioscuri della musa burlesca, sopraviveva il Firenzuola, appartato nel suo ritiro pratese, dove si sarebbe spento il 27 gingno I543, mentre a più lunga vita erano destinati Della Casa (m. I4 novembre I556), Bini (m. I554), Capilupi (m. Is60), Giova (m. post I569), tutti però variamente assorbiti da officia austeri, che li allontanarono per diversi percorsi dai «dilettevoli capricci» coltivati in casa Strozzi. Eccentrica la vicenda di Giova, il quale, in contatto con circoli valdesiani napoletani sin dagli anni Trenta, si orientò sempre più decisamente verso posizioni filoriformate e fini $i$ suoi giorni a Lione, dopo essere stato riconosciuto eretico dalla Repubblica di Lucca nel $1567 .^{4}$

Nel is4I la fama di Molza, anche se già affetto dalla grave forma di lue che ne limitò progressivamente la vita pubblica, travalicava l'orizzonte romano ed egli era noto e stimato nella società letteraria della penisola per la sua apprezzatissima produzione lirica latina e volgare. Se Sabino non aggiunse un nome prestigioso come il suo tra quelli dei contubernali burleschi dei conviti strozziani è da credere che lo facesse a ragion veduta, perché non considerava Molza membro del sodalizio.'

Non basta a rovesciare questa conclusione lo pseudonimo di Fico menzionato da Doni nella Libraria e nei Mondi accanto agli altri dello stesso conio adottati dai Vignaiuoli (Cotogno, Agresto, Palo, Viticcio, Cardo, Semenza, Borrana eccetera), nel quale si dovrebbe riconoscere l'appellativo grecizzante Siceo (da ov́xov "fico", per la sua particolare dolcezza), che è però attestato per Molža, come si vedrà più avanti, non prima del I538, un po' in ritardo sulle date dei Vignaiuoli. Soprattutto, come il nome di Vignaiuoli non trova conferma in altri documenti coevi, cosi pure per nessuno degli pseudonimi elencati da Doni si danno prove di un collegamento con i letterati della combriccola ricordata da 
Sabino. Senza contare che l'elenco di opere date ai Vignaiuoli nelpasso dei Mondi è inficiato da incertezze e anacronismi: la Cultivazione, il Dioscoride vulgare, la traduzione della Bucolica, il Comento, Lettere delle ville, gli Orti delle donne. Limitandoci ai due titoli identificabili con maggiore certezza, nel Comento va riconosciuto per antonomasia il celebre Commento di ser Agresto del Caro, che si lega alla Nasea dello stesso Caro menzionata da Doni poco dopo, opere andate a stampa nel I539, cioè più avanti rispetto alla breve stagione dei Vignaiuoli. ${ }^{6}$ Le Stanze di cultura sopra gli horti delle donne, cioè il Vendemmiatore di Luigi Tansillo, videro la luce in una stampa napoletana invito auctore del I534, di cui non ci sono pervenuti esemplari, poi a Venezia nel I537, ed esulano dalla esperienza del gruppo romano.

Percio, la duplice testimonianza di Doni, tarda rispetto agli eventi che descrive (la Libraria è del I550 i Mondi sono del I552) e poco spendibile sul piano dell'anagrafe e del catalogo delle opere, va presa come una costruzione a posteriori elaborata dall'estroso poligrafo, utile a ricomporre la memoria lasciata dall'esperienza romana, piuttosto che a ricostruirne la realtà storica. ${ }^{7}$

Esitate a vuoto le testimonianze letterarie, per collegare Molza con qualche garanzia in più alle attività del sodalizio vignaiuolo soccorrono i documenti epistolari, da soppesare però con cura. Il I2 aprile I534 Berni, scrivendo a Giovanfrancesco Bini da Firenze, chiude l'epistola con $i$ saluti da presentare agli amici romani e con un protratto angurio giocoso diretto al destinatario, declinato sullinvenzione priapesca, che era stata alla base del primo dei due osceni capitoli burleschi dedicati da Bini alla lode dell'orto (il secondo risale all'anno dopo):

quando vi vien visto monsignor di Segni, alias monsignor Grana, [vorrei che] vi piacesse fare a sua signoria le mie umili raccomandazioni: e poi di mano in mano alli altri signori, come vi piace; e, fra gli altri e sopra gli altri, al dabenissimo signor Molza, a messer Giovanni della Casa, e tutta quella divina academia. Così vi dia Dio grazia d'avere un priapone grande per il vostro orto, con una fascina trabale fra $<$ le $>$ gambe, et una falciazza in mano, e che non vi si accosti mai né brinata né nebbia né bruchi né vento pestilente, e abbiate fave e baccelli e pèsche e carote tutto l'anno, sì come desidero di avere io nel mio orticciolo fallito qua giù, che attendo pure a raffazzonarlo quanto posso. ${ }^{8}$ 
La lettura di queste righe che è prevalsa finora ha visto identificare l'accademia evocata da Berni con il cenacolo di ispirazione priapesca e orticola, del quale il capitolo di Bini si offre come prodotto esemplare, dunque con $i$ Vignaiuoli, ai quali sono stati affiliati anche $i$ due letterati nominati appena prima, cioè Molza e Della Casa. Ora, preso nel suo insieme e sottoposto a un esame giudizioso, il passo si presta a uno scioglimento differente. Il vescovo di Segni che inaugura la lista degli omaggiandi è Lorenzo Grana, creato presule di quella diocesi da Clemente VII il 3 gingno I528 e più tardi segretario ai brevi sotto Paolo III (mori il s settembre I539), noto per avere pronunciato l'orazione funebre di Egidio da Viterbo nel I532, ${ }^{9}$ persona, si può dare per certo, aliena da sentimenti burlevoli. Di seguito, l'invito ad estendere $i$ saluti agli altri conoscenti romani coinvolge i nomi dei due letterati più illustri, gratificati dei titoli di signore e messere, Molza anche di un elativo che gli dona ulteriore spicco, e infine "quella divina academia», per la quale, se si vuole pensare che sia il cenacolo dei cosiddetti Vignaiuoli, si è obbligati a interpretare l'aggettivo in maniera antifrastica e considerarlo una spiritosaggine sui contenuti della loro poesia, di cui tutto si poteva dire meno che fossero divini. ${ }^{\text {IO }}$

Se ci si attiene al livello letterale, «divina» è nella epistola l'aggettivo qualificativo che individua il circolo dei letterati romani nel suo complesso, a cui si ascrivono Grana, Della Casa e Molza, cioè tre cultori dell'oratoria e delle Muse latine che perpetuavano la tradizione della gloriosa Accademia Romana, quella, infine, per la quale si poteva adoperare il nobile vocabolo di ascendenza platonica, sproporzionato, quando scriveva Berni, per l'informale conventicola dei seguaci della sua maniera di poetare attivi nell'Urbe. Altro significato aveva assunto il termine all' altezza in cui scriveva Doni, quando poteva essere impiegato con proprietà per indicare i fenomeni di socialità letteraria diffusi nella penisola in forme regolate. All'accademia dei letterati romani nel loro complesso, come si conviene alla gerarchia discendente dei saluti, si contrappone nella lettera di Berni il protratto augurio rivolto in chiusura al sodale di un'altra esperienza, avvertita come congeniale, tanto da innestare un'impennata stilistica nella testura dell'epistola, ma più umile e dimessa del tono adoperato per $i$ tre letterati di ascendenza classicistica prima citati.

Nelle missive di Berni in questo periodo estremo per lo sfortunato letterato fiorentino il nome di Molza, talora affiancato a quello di Della 
Casa, emerge sempre aureolato da un riguardo ossequioso che mette in guardia dal reclutamento sic et simpliciter del modenese nei ranghi del cenacolo il cui prototipo era la poesia priapesca di Bini. Cosi lascia intendere la lettera a Carlo Gualteruzzi del 29 settembre Is34: «Né mi occorre altro da dirvi se non che vi prego mi amiate e teniate in grazia di quelli che sapete desidero che mi diano la grazia loro, e particularmente del mio messer della Casa e del Molza, se però gli cale mirar si basso, ecc.». E il 3 I marzo I535: "Raccomandatemi a monsignor de' Carnesecchi, a messer Giovanni della Casa et al Molza; e voi amatemi». E ancora, il 7 maggio I535, nell'ultima lettera, scritta a pochi giorni dalla fine, la testimonianza in apparenza più invocabile per includere Molza nelle fila dei Vignaiuoli, se non fosse che egli verrebbe a trovarsi nella posizione di loro membro piu rappresentativo, al punto di essere menzionato lui per tutti, e cio risulterebbe in contraddizione con quanto emerso dai frammenti fin qui citati:

Vi prego, tenetemi in grazia del signor messer Giovanni della Casa e diteli che io sto per affrontarlo di questo suo bel luogo che ha qua presso alla loggia de' Pazzi, se non credessi che fusse per tornare a Firenze, come vorrei però che facesse e lo desidero. Raccomandatemi al Molza et a tutta la academia. ${ }^{\text {II }}$

Sorvolando sulla parte relativa a Della Casa, di pertinenza fiorentina, la raccomandazione finale, nella sua generica asciuttezza, non impedisce di considerarla di nuovo diretta all'insieme dei letterati frequentati da Berni negli anni romani e non alla accolita più ristretta dei cultori della sua maniera, per definire la quale egli era ricorso nella lettera a Bini a una forte caratterizzazione. ${ }^{22}$

Berni si trovava a Firenze dal settembre I533, quando aveva interrotto con un pretesto il viaggio al seguito del cardinale Ippolito de' Medici, che si recava a Nizza ad assistere alla formalizzazione dell' accordo sulle nozze tra la nipote del pontefice, Caterina di Lorenzo duca d'Urbino, e il delfino Enrico di Valois. Rientrato Ippolito a Roma, Berni continuò a resistere alle ingiunzioni del porporato, che lo reclamava accanto a sé. Dall'epistolario risulta che l'insistenza di Ippolito aveva assunto toni accesi ed è probabile che Molza, come segretario e confidente del Medici, avesse parte nel carteggio intercorso tra quest'ultimo e il letterato fiorentino, che non ci è giunto, così come, è evidente, non ci sono 
giunte missive personali scambiate tra $i$ due poeti. Epperò da un passaggio della lettera a Gualteruzzi del 6 febbraio I535 si ricava che Berni richiese a Molza il testo della violentissima orazione pronunciata in Campidoglio nell'estate I534 contro Lorenzino de' Medici, reo di avere decapitato in una scorribanda notturna le statue dell' arco di Costantino $e$ in altri luoghi di Roma, in un gesto risultato tanto inviso all'ambiente dell'Urbe, intriso di culto della romanità, quanto tuttora oscuro nelle sue motivazioni. Il passo va ad aggiungersi alle testimonianze epistolari esaminate sopra: "Vi prego, tenetemi in grazia di monsignor de Carnesecchi e di messer Giovanni della Casa e del mio dolcissimo Molza, non obstante che non mi voglia compiacere di quella oraziones. ${ }^{{ }^{3}}$ Il capitolo a Sebastiano del Piombo, forse del settembre I534, l'ultimo composto da Berni, lascia intendere che alla consuetudine degli anni romani, se non altro per il servizio prestato da entrambi a Ippolito (assai fugace, in verità, quello di Berni), non subentrò uno stabile commercio epistolare, per ragioni che Berni, con una comica ma non per questo finta deminutio, imputa alla propria inadeguatezza culturale:

Fatemi padre, ancor raccomandato

al virtüoso Molza gaglioffaccio,

che m'ha senza ragion dimenticato;

senza lui parmi d'esser senza un braccio:

ogni dì qualche lettera gli scrivo

e perché l'è plebea da poi la straccio. ${ }^{\mathrm{I4}}$

Nell'espressione colorita del $v .76$ che esprime la nostalgia per il sodale romano non è da vedere più dell'ossequio al codice comico, che imponeva iperboli espressive di questo tipo: Berni ricorda qui l'affabile cortigiano e il conversatore faceto, non il contubernale burlesco. Una preziosa spia linguistica è invece il termine gaglioffaccio, che faceva parte del lessico caratteristico di Molza, come ci ricorda Varchi, che lo aveva conosciuto bene, nell'Hercolano:

VArChi. Nè anco messer Bernardino Daniello, che fu l'anima di messer Trifone Gabriello, come era messer Trifone Bencio nipote di ser Cecco, senza 'l quale non poteva stare la corte, di quel gaglioffaccio del Molza. Conte. Come, gaglioffaccio?

VArchi. Gaglioffaccio nell'idioma del Molza significava huomo buono e da bene. $^{\text {Is }}$ 
Tramite questa dialogicità, dal capitolo berniano si affaccia cosi l'immagine di un Molza sorridente e motteggevole, cui è consacrata la memoria sua più serena degli anni romani, lontana dai rovesci della carriera cortigiana, dalle vicissitudini familiari, dalla malattia profligante.

Che Molza, preso dai giri mondani e dalle avventure sentimentali, non curasse la corrispondenza con l'amico lontano è più che verosimile. Cade però in questo periodo il sonetto Berni, sei tristo o lieto? temi o spere, esempio qualitativamente alto di corrispondenza in versi, di cui Molza ci ha lasciato più di un saggio tra le sue rime (meglio noti sono gli scambi intercorsi con Pietro Bembo e con Vittoria Colonna):

Berni sei tristo o lieto? temi o spere danni aspri e gravi, o gloriose prede, poich'a Vienna il Turco ora non siede, né d'intorno accampa sue infinite schiere?

Qui più diletta assai false, che vere novelle udir, ma io do maggior fede a quella parte, che per fermo crede che pianger debbia Italia in vesti nere;

or che lo tuo mortal nel loco alberga che li diè vita in prima, e i sette colli non vede, e più tra lor carte non verga, tenga giusto dolor gli occhi tuoi molli, perché l'anima al ciel devota s'erga, da sé sgombrando i pensier bassi e folli. ${ }^{16}$

L'interrogativo sulla pressione turca in Occidente con cui si apre il sonetto riporta al I534, quando ci si poteva augurare un alleggerimento in ragione della guerra portata da Solimano I alla Persia, ma non oltre il mese d'agosto, quando ebbe luogo l'incursione del corsaro Khair ad-din detto Barbarossa, il quale, sbarcato vicino a Gaeta, tentò invano di rapire Giulia Gonzaga per offrirla in dono a Solimano I. La duchessa di Fondi si mise in salvo per un soffio e Ippolito de' Medici si precipitò a soccorrerla alla testa di un manipolo di armati. Impossibile che dopo il clamore suscitato dall'evento Molza si ponesse a scrivere quei versi pensosi all'amico lontano.

Dimidiato tra il persistente pessimismo sulle sorti della penisola e riflessioni private, è in questo secondo versante che risiede l'importanza del componimento. Nel monito penitenziale rivolto all' amico affinché si 
dedichi a una nuova maniera di poesia dai contenuti, cosi si dice esplicitamente, devoti, non vedo infatti cosa altro si debba vedere se non l'espressione di un amabile ma sostanziale dissenso dalla maniera satirica e burlesca alla quale Berni aveva consacrato la sua musa e dalla quale era sollecitato ora a prendere le distanze, quasi il ritorno in patria offrisse la chance di rinunciare alle blandizie e alle vanità della metropoli pontificia per ritrovare una genuina e salutare vena poetica. Molza indossava cosi l'abito del moralista, che non gli era proprio, e a fatica poteva rimuovere il rapporto di complicita che si era stabilito tra $i$ due letterati negli anni romani. Una testimonianza di ciò viene dal capitolo a messer Baccio Cavalcanti Sopra la gita a Nizza, che mette in burla le smanie di prelati e gentiluomini accorsi in massa all'evento svuotando l'Urbe. Alla fine del primo dei due capitoli metricamente distinti in cui si articola l'epistola in versi (vv. 82-94), Berni introduce Molza che risponde per le rime alla battuta grassoccia di un cortigiano a proposito del rischio di finire catturati da Barbarossa durante il viaggio e subire il supplizio dell'impalazione:

Mentre scrivevo queste cose mi sovenne del Molza nostro, che mi disse un tratto un detto di costor molto solenne:

fu un che disse: «Molza, io son sì matto, che vorrei trasformarmi in una vigna, per aver pali e mutarli ogni tratto.

Natura ad alcun mai non fu matrigna: guarda quel ch'Aristotel ne' Problemi scrive di questa cosa»; e parte ghigna.

Rispose il Molza: «Adunque mano a' remi; ogniun si metta dietro un buon temone et andiam via, ch'anch'io trovar vorre'mi

a così glorïosa impalazione». ${ }^{17}$

Berni colloca l'aneddoto alla fine della sua perorazione contro l'opportunità di intraprendere il viaggio, come emblema della insana follia, ma anche del vizio ad essa sottostante, che pervade ai suoi occhi la Curia pontificia e per estensione il mondo della corte in generale. Qui si avverte la distanza tra il fastidio maturato da Berni verso quella realtà e l'atteggiamento di Molia, che invece ne fu spettatore integrato e solerte interprete, incapace di intravederne il superamento in una dimensione 
altra sia sul piano politico sia su quello estetico, ma anche di avvertire un disagio sostanziale per $i$ rituali e le convenzioni che la caratterizzavano. Il suo distacco non andò oltre un signorile anticonformismo e una bonaria ironia, che incantava coloro che lo frequentavano, ma che non si tradusse in giudizio moralistico, come mostra la sorridente canzonatura opposta alla dichiarazione di pederastia (con tanto di legittimazione filosofica) del cortigiano romano. È qui, probabilmente, che si deve individuare il limite della sintonia di Molza con il clima morale che riuniva $i$ cosiddetti Vignaiuoli intorno all'adozione della formula bernesca. In essa, iusta la lettura in profondità di Romei, si deve intravedere il malessere di una generazione di poeti che con il trauma del Sacco aveva vissuto la fine di una letteratura incentrata sul culto fuori dal tempo di una classicità imperitura, e che nell' accogliente rifugio di una satira di modesta temperatura polemica, o nel culto del paradosso burlevole tentava di esorcizzare l'incertezza sulle vie da intraprendere per costruire una nuova identità dell'nomo di lettere e dei suoi rapporti con il potere.

Inutile dire che nel Is33 Molza, a differenza di Berni, non si poté esimere - né si può immaginare lo desiderasse - dal seguire Ippolito a Nizza, sebbene la conferma della sua presenza sia legata per noi alla labile testimonianza, appena un cenno, di una lettera di Giovanni Della Casa a Carlo Gualteruzzi, da Firenze il 2 gennaio I534, di cui, in mancanza di meglio, siamo costretti ad accontentarci. Il futuro monsignore scherza su quanto Gualteruzzi, che aveva seguito la Curia nella trasferta transalpina, gli doveva avere riferito sull'intraprendenza del comune amico con il gentil sesso: "Il Molza ha preso un brutto costume in Francia a voler basciar le donne: li sarà pelato la barba e forse altro. Lo saluto molto molto». ${ }^{\mathrm{I}}$

Se si tira il bilancio della documentazione che abbiamo raccolto fin qui, si è tenuti a riconoscere che, oltre a non filtrare a una data alta indizi di un effettivo cimento di Molza nell'esercizio delle Muse burlesche, si deve dare anche una valutazione prudente sulla sua collateralità al gruppo dei Vignainoli, che si riduce, per quanto ci è dato sapere, al rapporto che lo legò a Berni, ma su un piano diverso, si è visto, dalla condivisione di una maniera di poetare e del sentire che ne costituiva le premesse. ${ }^{\text {I9 }}$

Di «una relativamente tardiva conversione del Molza [...] dalla "gravità" umanistica alle "baie" della poesia bernesca» ha parlato Romei2 
sulla base della presentazione che Caro fa del poeta modenese in principio del Commento di ser Agresto sopra il Capitolo dei fichi moliziano:

Il Padre Siceo non entrò egli per questa via del Bernia, percioché s'era concio prima con Apollo per iscrivano delle faccende del Mastro di casa, e si stava in su la gravità con le Muse, perché s'arrecavano in contegno con esso lui. Ma, poi che vennero questi buon compagni, e s'avvide che le Muse ancor elle volevano il giambo, si mise in frotta con loro a fare ancor esso delle baie. E così scrisse dell'Insalata; scomunicò le Scomuniche. ${ }^{21}$

Il passo serve, naturalmente, in primo luogo a collocare anche gli altri due capitoli, sulla insalata e la scomunica, oltre a quello oggetto del Commento, a prima del termine certo dell'agosto I539, data di stampa dell'operetta, arretrando con tutta probabilità almeno al principio del I538, quando risalirebbe la prima stesura del Commento. Per il Capitolo dei fichi si dà un termine nella diceria sulla Corona di gramigna, presentata da Pietro Paolo Gualtieri a Claudio Tolomei nel suo settimanale Regno della Virtù durante il Carnevale del I538, nella quale Bacco è detto cinto «d'edera (o di fico, come piace al nostro padre Siceo)», ${ }^{22}$ con riferimento al v. 25 del capitolo, dove, appunto, Bacco è «cinto di fichi il crin».

Altra inferenza che si ricava dal passo cariano è che il Capitolo dei fichi è da posporre cronologicamente agli altri due, dato in qualche modo confermato anche nella tradizione a stampa, che vede la princeps dell'Insalata $e$ della Scomunica nelle Terze rime del Molza, del Varchi, del Dolce, et d'altri, Venezia, C. Navò et fratelli, I539, distinta, sebbene quasi simutanea (probabilmente anteriore), a quella dei Fichi nella citata edizione romana del I539. Tutti e tre $i$ capitoli sono però accomunati dall'avere come destinatario Trifone Benci, del quale si fa menzione in essi con minore o maggiore insistenza (Della insalata, v. I3; Della scomunica, vv. I, 37, 46, 70; Dei fichi, vv. 97, 205) e nel Commento di ser Agresto è presentato come «un nomo perfetto amico del nostro Poeta». ${ }^{23}$ Per quanto sappiamo della biografia di Benci, ${ }^{24}$ egli era a Roma nell'aprile I535 con un ufficio di segretario nella Cancelleria apostolica ed è probabile che il suo arrivo nell'Urbe dalla natia Assisi sia coinciso con il principio del papato farnesiano, alcuni mesi prima. ${ }^{25}$ 
Divenuto intimo di Molza, alla fine del I539 era lui ad assisterlo in una delle prime fasi acute del suo male. ${ }^{26}$ Nel febbraio I537 fu aggregato al seguito del cardinale Reginald Pole legato in Francia e nei Paesi Bassi, con il fine di indurre Inghilterra e Scozia a ribellarsi a Enrico VIII, e rimase lontano da Roma fino all'autunno. ${ }^{27} \mathrm{Di}$ nuovo, al principio del IS4I segui Gasparo Contarini inviato alla dieta di Ratisbona e rimase alle sue dipendenze fino alla morte del porporato (a Bologna il 24 agosto I542); poi si ricongiunse con Molza dopo che questi, molto malato, si era ritirato a Modena (maggio I543), per stargli accanto pemurosamente fino alla fine. ${ }^{28}$

Se si tengono presenti queste date e si dà fede cronologica a quella che potrebbe anche essere la celia di Caro verso il contegno sussiegoso dell'amico, raffinato poeta latino e rimatore elegante, ritroso a scendere sul terreno burlesco, risulta la compressione della esperienza molziana in questo campo in un volgere abbastanza breve, collocato tra le testimonianze che si sono esaminate intorno alla conventicola dei Vignaiuoli, con le quali arriviamo alla metà del I535, e il Commento cariano. Un lasso all'incirca di due anni e mezzo, il cui inizio viene a coincidere a un dipresso con l'evento traumatico della morte di Ippolito de' Medici (Io agosto I535). Molza depose allora la vita brillante e movimentata condotta accanto all'antico padrone e risiedette a Roma (con alcune pause: tra l'agosto e il novembe I536 era a Modena), privo di ufficio e di un punto di riferimento preciso all'inizio del pontificato farnesiano, nel quale subiva, come satellite del Medici, una inevitabile emarginazione dagli ambienti politici e diplomatici più prestigiosi e probabilmente anche un temporaneo oscuramento dalla vita letteraria dell'Urbe, di cui era stato fin li protagonista. A ciò si aggiunsero le angustie economiche e la delusione subita per il fallimento dei progetti matrimoniali del figlio Camillo con Ludovica di Antonio Colombi, nipote del facoltoso banchiere modenese Ludovico Colombi, per i quali nel corso del Is 37 Molza si spese con assiduità, sollecitando le sue conoscenze altolocate.

Alliniziativa di Paolo Sadoleto, a Nizza, dove si trovava al seguito dello zio Iacopo per il congresso tra l'imperatore e il re di Francia patrocinato da Paolo III nel gingno I538, si deve l'intervento del cardinale modenese presso il pontefice, che al ritorno della Curia a Roma procurò al poeta l'assunzione al servizio del nipote, il giovane cardinale Alessandro Farnese. Presso di lui Molza tornò in migliore stato ed 
entrò a far parte da una posizione di primo piano della cerchia gravitante intorno al colto e intelligente porporato, che era la condizione a lui congeniale come nomo e come letterato, incapace di intravedere una alternativa che consentisse l'esercizio delle lettere affrancato dal servizio cortigiano. Inangurò cosi il nuovo corso di letterato farnesiano, che lo concentrò subito in una fitta produzione encomiastica latina, mentre l'esercizio poetico in lingua si orientò docilmente a condividere temi e modalità con i poeti della cosiddetta Accademia Farnesiana.

I tre esemplari burleschi molziani - dei fichi, dell'insalata, della scomunica - si trovano dunque paradossalmente incastonati nel periodo più triste e sconsolato dell'esistenza del letterato, fatta salva la fase ultima della malattia, in un momento di incertezza materiale sul proprio destino e di interiore ripiegamento dovuto alla fine del rapporto anche ideale e affettivo che si era stabilito con Ippolito. Si trattava di una congiuntura delicata e dolorosa, in cui Molza, dopo il tracollo dell'esperienza cortigiana e alle prese con le deludenti vicissitudini familiari, era forzato a cercare una nuova immagine privata e pubblica individuando nuovi mecenati e aprendosi a punti di vista inediti anche nella pratica letteraria. In questa temperie è verosimile che il poeta modenese sia stato coinvolto dagli amici romani più cari nelle loro lepidezze poetiche, con l'intenzione di risarcirlo delle delusioni subite e di recuperarlo a una vita di società.

Dissoltasi a una data che non è possibile precisare a causa del suo carattere informale l'esperienza dei Vignaiuoli, la genesi del cenacolo che si diede il nome di Gioco o di Reame della Virtù non è ricostruibile con certezza, né ci sono elementi per pensare a una filiazione o per lo meno a una continuità diretta con il precedente sodalizio. Nel Ragionamento sopra alla proprietà delle imprese Luca Contile indica una nascita singolarmente precoce, laddove riferisce che la Virtù aveva eletto suo protettore Ippolito de' Medici, ma il cardinale era mancato proprio mentre era in corso la ricerca di un'impresa in cui compendiare il programma dell'Accademia e cosi fu deciso di rinunciarvi, argomentando che non era più possibile dare una rappresentazione della Virtù poiché essa si era spenta insieme con Ippolito. ${ }^{29}$ La suggestiva notizia trova forse conferma in un passaggio della Formaggiata di sere Stentato al Serenissimo Re della Virtude, opera di Giulio Landi databile al I538-39. Alla fine della premessa A' lettori a firma dello stampatore Ser Grassino è ricordato «quello 
virtuosissimo et magnanimo Signore Hippolyto Cardinale de' Medici, padre de' virtuosi, et di tutte le virtuti vera base et fermo sostegno», ${ }^{3 \circ}$ ma è evidente che l'elogium potrebbe anche valere in universale e non riferirsi espressamente ai Virtuosi romani.

In effetti, $i$ dati certi che possediamo sulla Virtù convergono tutti tra l'inverno e principio della primavera I538. Come è noto, l'attività dell'Accademia coincideva con la durata del Carnevale, cioè tra l'Epifania e l'inizio della Quaresima, durante il quale veniva eletto a sorte un re - detto "re della fava" dall'antica modalità del sorteggio, consistente nel nascondere una fava nell'impasto di una torta, e incoronare colui al quale toccava -, il cui regno aveva durata settimanale e al quale gli altri membri presentavano doni scherzosi, accompagnati da componimenti burleschi.

La produzione dei Virtuosi in versi e in prosa si ricostruisce grazie ai testi trasmessi dalle Lettere facete di Dionigi Atanagi e di Francesco Turchi, antologizzati da Bartolomeo Gamba nelle Dicerie di Annibal Caro e di altri a' Re della Virtù (Calvely-Hall [Venezia, Alvisopoli], I82I), e, in forma più genuina, a una preziosa antologietta di componimenti in un quaderno legato nel ms. MM 693 della Biblioteca civica di Bergamo, di cui parla in queste stesse pagine Enrico Garavelli e percio rinvio senz'altro al suo contributo.

La presenza di Molza sin dalle prime riunioni è accertata dalla testimonianza di Caro, che il Io gennaio scrive a Benedetto Varchi: "Questa sera [Molza] sarà qui a cena, ché faremo il reame della befania, dove saranno di molti passatempi». ${ }^{31}$ Il 10 marzo, sempre a Varchi, Caro comunica con entusiasmo il successo dell'iniziativa: "Il Giuoco de la Virtù crebbe tanto che diventò e questa settima si son fatte gran cose», diffondendosi sulle modalità delle riunioni. ${ }^{32}$ Finché due lettere del Io aprile comunicano non tanto la repentina inspiegabile catabasi del fervore, quanto semplicemente l'avvento della Quaresima, che poneva fine alle riunioni conviviali per quell'anno: a Bernardino Maffei Caro scrive quel giorno che «il Regno de la Virtù è sbandato»e a Giovan Francesco Leoni che "Il Regno de la Virtù è in declinazione»». ${ }^{33}$ I tre capitoli di Molza, secondo la cronologia che abbiamo ricostruito, precedono di poco la fioritura della Virtù e il loro metro lungo, molto differente dalle stanze con cui gli accademici accompagnavano gli omaggi giocosi al re di turno, non li rendono spendibili nei rituali carnevaleschi dei Virtuosi, cosi come pure non v'è traccia in essi dell'esibizione della 
socialità che emerge invece in forma molto esplicita dai componimenti degli altri Padri dell'Accademia. Postumi all'esperienza dei Vignaiuoli, i capitoli di Molza rappresentarono il frutto tardivo e qualitativamente alto di quella prima stagione di poesia comica nella Roma posteriore al Sacco. Un frutto cooptabile agli occhi dei nuovi cultori romani del genere nel modello di comico colto, di cui la Virtù si proponeva di essere la levatrice proprio a partire dall'abbandono di una pedissequa riproposta della formula bernesca, già allora orientata verso un riflusso formulare e autoalimentato, incapace di aperture verso altri apporti e di contaminazioni con il mondo classico, cui tra $i$ Virtuosi almeno Caro $e$ Tolomei guardavano con lucida progettualità. Il cariano Commento di ser Agresto sul Capitolo dei fichi di Molza rappresenta l'esempio più eclatante, e destinato a formidabile successo, dell'acquisto al nuovo programma della formula bernesca, recepita nell'esemplare reputato più congeniale a un'idea di comico come terreno aperto a contaminazioni fra tradizione volgare ed eredità classica, aneddotica ed erudizione, quotidianità e raffinati intarsi antiquari, paradigma filologico ed espressività popolare, quale si dà nel Commento. ${ }^{34}$

A proposito del profilo alto degli specimina burleschi di Molza, si deve evocare qui il bilancio critico, remoto nel tempo dagli eventi che stiamo illustrando, formulato da Benedetto Varchi, poiché va esattamente nella nostra direzione. Prima nella Lezione della poetica in generale, letta nell' Accademia Fiorentina la seconda domenica d'ottobre I553, e poi nel Quesito ottavo dell'Hercolano ( $D$ a chi si debbano imparare a favellare le lingue, o dal volgo, o da' maestri, o dagli scrittori») Varchi accampa a proposito della poesia ridicola una pregiudiziale di naturalezza che mette $i$ fiorentini in uno stato di obiettivo vantaggio sugli altri cultori del genere, incapaci di produrre una poesia burlesca genuina e di essere immuni da contaminazioni con i generi seri. Il giudiz̨io è ricco di sfumature, ma qui interessa evidenziare che la qualitas del Molza bernesco è individuata da Varchi nel surplus di erudizione, che lo colloca su un piano diverso dalla naturalezza spontanea che richiede questo genere di poesia. Cosi nella Lezione della poetica in generale:

Al Molza ancora, che ingegnosissimo era e dotto, ed esercitato molto, non venne fatto esser poeta burlesco con tutto che provasse. Ed io porto ferma opinione, che chi non è nato in Firenze, o almeno stato in Firenze 
assai, non possa in questo genere divenire eccellente; il quale però credo che sarebbe stato bene, che come col Berni nacque, e da lui si noma, e per lui si figura, così con lui si fosse ancora spento. ${ }^{35}$

\section{E cosi nell'Hercolano:}

Conte. I capitoli del Mauro e quegli d'alcuni altri sono pur tenuti molto dotti e molto begli.

VARCHI. Già non si biasimano per altro, se non perché sono troppo dotti e troppo begli; e in somma non hanno quella naturalità e fiorentinità (per dir cosi), la quale a quella sorte di componimenti si richiede. Messer Mattio Franzesi, mio amicissimo, avanzò tanto il Molza nello scrivere in burla, quanto il Molza, che fu non meno dotto e giudizioso che amorevole e cortese, avanzò lui nel comporre da buon senno. ${ }^{36}$

Ai conviti dei Virtuosi non partecipò Mattio Franzesi, con suo grande rimpianto. Nel gennaio-febbraio I538 cade infatti il viaggio che lo portò a Padova e a Venezia, ${ }^{37}$ dove si sarebbe trattenuto almeno fino all'estate. Lo scopo, o almeno uno degli scopi, era di portare a Benedetto Varchi il manoscritto della Ninfa Tiberina, finalmente terminata da Molza; già il I2 febbraio Franzesi descrisse a Molza la lusinghiera accoglienza ricevuta dall'opera negli ambienti letterari veneti e il 6 marzo successivo riferi la reazione partecipe del Bembo alla Ninfa $e$ agli altri componimenti latini e volgari molziani che egli aveva portato da Roma. ${ }^{38}$ In una serie di quattro capitoli, appunto D'un viaggio, Franzesi forni una descrizione dettagliata dell'itinerario attraverso il Lazio, l'Umbria, la Marca, la Romagna, fino a Bologna, Ferrara e a destinazione, secondo la consolidata topica oraziana dell'iter pessimum. Il primo di essi, Com'io partii, da voi, con voi rimasi, a Benedetto Busini, illustra le tappe iniziali lungo la via Flaminia, appena lasciata l'Urbe. La mente del viaggiatore corre ai ludicra che si celebravano allora nella Virtù e vi troviamo un bel catalogo dei protagonisti, tra di essi il Molza, distinto dall'appellativo che era divenuto una sua prerogativa. Degli altri, riesco a identificare (con cautela) solo il primo, nominato anche nella Statua della Foia di Caro, ${ }^{39}$ che dovrebbe essere il pittore Sebastiano del Piombo:

Pur venni a Castel Nuovo, et non mi volli fermarvi, et passai via verso Rignano col pensier ritornando a i sette colli, 
che mi sovvenne all'hor di fra Bastiano, che questa sera assiso in maestà, honorerà la Fava a piena mano, et con perfetta sua divinità il purpureo Rossello, et Grassellino, et il sugnoso Lencio humetterà.

Vedo Messer Ferrando, et Messer Bino, il Cidalgo, l'Ancona, il Ticco, e 'l Caro, il Tolommeo, e 'l Molza arcidivino; che con leggiadro stile ornato et chiaro del re canta le lodi; et n'è ben degno il pennel dotto e 'l suo disegno raro; tra gli altri ancora il gentil Fabio Segno fia forse stato, et voi 'nsieme, ma vui havevi però fatto altro disegno.

Hor, mentre l'alma pensa a tutti i sui cari amici et padroni, a Rignan giunsi in su 'l scoccar de' tocchi ventidui. ${ }^{\circ}$

Giunto a Spoleto, Franzesi si concede un sonno «sodo sotto un cortinaggio», ma tra il sonno e la veglia il pensiero va ancora agli amici romani, dei quali solo Molza merita una menzione, posta a sigillo dell'epistola in versi:

Voi (se' dí vostri sien sempre felici) dite al Molza di gratia per mia parte, et scongiuratel per le Beatrici,

che si degni tal'hor di farne parte de le divine sue compositioni, mandatele dipoi con vostre carte;

e qui fo punto, e affibbiovi gli sproni. ${ }^{4 \mathrm{I}}$

Ancora nel nome di Molza si apre il capitolo III, diretto ad Annibal Caro, dedicato al tratto da Loreto a Bologna:

Caro mio caro, io so che voi sapete a i quanti dì è san Biagio, et perch'io 'l dica, poco di sotto ve ne accorgerete.

Che fa tutta la schiera nostra amica di casa e fuore? il signor Molza nostro come corteggia e come s'affatica 
per celebrare con sì purgato inchiostro il suo sacro signore? et com'è in gratia di quella che per gratia il ciel gli ha mostro? ${ }^{42}$

Il sacro signore per cui Molza versa il suo inchiostro purgato (a differenza di quello impuro di Franzesi e dei sodali a cui egli scrive) non può che essere il cardinale Alessandro Farnese e nella dama vagheggiata dal poeta si deve riconoscere Faustina Mancini, la giovane nobildonna celebrata dai poeti dell'Accademia Farnesiana per la sua bellezza e per le sue virtu, andata sposa del condottiero Paolo Attavanti il 28 febbraio 1538 e destinata a morire di parto il 6 novembre $1543 .{ }^{43} \mathrm{La}$ presenza di Farnese nel ternario come padrone di Molza ha rilevanza a fini cronologici: il poeta entrò infatti al servizio del prelato nella seconda metà del I538, dopo il ritorno della Curia a Roma dallincontro di Nizza tra Carlo V e Francesco I (Paolo III rientrò nella sua residenza il 24 luglio) e dunque $i$ capitoli odeporici di Franzesi sono da posporre a questa data. Al contempo, evidentemente, presentare Molza nella cerchia di Farnese già all'inizio dell'anno costituiva un anacronismo, cui Franzesi si prestò perché quando compose i capitoli Molza era ormai consacrato nel suo ruolo di letterato farnesiano.

La devozione di Franzesi per Molza che emerge dai capitoli si spiega, come si è detto, con il fatto che il fine della sua missione era appunto la presentazione dei componimenti del poeta in Veneto, in circoli del massimo prestigio. Ciò coronava l'attività svolta negli anni precedenti, lungo $i$ quali Franzesi aveva tenuto informati $i$ suoi corrispondentiVarchi primo fra tutti, ma anche Pietro Vettori a Firenze - sulla produzione molziana e, vincendo la ritrosia del poeta, aveva ottenuto di trarre copia delle sue poesie, svolgendo cosi un ruolo importante nella diffusione delle sue opere a quella altezza. ${ }^{44}$ Nonostante la consuetudine, il carteggio di Franzesi accerta che non venne mai meno da parte sua il tono di ossequiosa deferenza nel trattare con Molza. La citata lettera da Padova del I2 febbraio I538, ad esempio, si conclude con una formula che tradisce un rapporto da inferiore a superiore: "Vostra signoria - termina la lettera - si degni tenermi vivo nella sua grazia, e si ricordi di me quando dà fuore alcuna divina sua composizione». ${ }^{45}$ Non so se sia questa condizione sufficiente per revocare in dubbio l'attribuzione a Franzesi dei due capitoli Contra lo sberrettare (inc. Messer Molza che sì, s'io me la incapo) $e$ Contro il parlar per 
Vostra Signoria (inc. Nel tempo che quest'era un'altra Roma), dati all'autore toscano nel secondo volume delle Opere burlesche, stampato dai Giunti di Firenze nel I5ss (rispettivamente cc. 62r-64v e 6rr-7Ir). Contra lo sberrettare era però già andato a stampa nelle Terze rime del Molza, del Varchi, del Dolce, et d'altri (Venezia, C. Navò et fratelli, I539, cc. $\left.A_{V I} v-B_{I} r\right)$, con l'attribuzione al pistoiese Paolo Panciatichi. Che $i$ due capitoli siano da assegnare a un solo poeta lo prova la quinta terzina di Contro il parlar per Vostra Signoria, che contiene una espressa indicazione in merito ("Onde quanto l'usanza sia molesta [scil. lo sberrettare] / vi dissi, Molza, in quella berta mia, / alla qual, per sorella io vo dar questa»). Essi insistono sull'indifferenza all'etichetta, che abbiamo detto essere stato tratto saliente dello stile di comportamento del letterato modenese e che l'autore sviluppa interpretando in maniera piuttosto convenzionale il fastidio per $i$ rituali cortigiani, che era tra $i$ contenuti piu autentici $e$ insieme facilmente ricevibili del bernismo. Ciò che lascia un po' esitanti circa la loro assegnazione a Franzesi è lo schietto cameratismo ostentato nei ternari, che porta a prendere in considerazione la candidatura di un letterato certamente più disinvolto come Panciatichi, cultore delle rime del Cammelli e disinibito verseggiatore burlesco, ${ }^{46}$ sul quale, in verità, l'epistolario molziano restituisce nulla più che la conoscenza e per un periodo molto ristretto (il luglio-agosto I535, poco prima della morte di Ippolito de' Medici), ma è legittimo immaginare una consuetudine di rapporti. ${ }^{47}$

A non molta distanza dagli eventi che stiamo narrando si colloca la già citata Formaggiata di sere Stentato al Serenissimo Re della Virtude, opera di Giulio Landi, apparsa a stampa «in Piasenza per Ser Grassino Formaggiaro MDXLII», ma assegnata da Dennis Rhodes ${ }^{48}$ a Gabriele Giolito, scartando la candidatura, avanzata a suo tempo da Gaetano Poggiali e ripresa da Bartolomeo Gamba, di un'iniziativa tipografica di Antonfrancesco Doni, allora soggiornante a Piacenza. Massimo Baucia ha individuato nel testo il termine post quem del soggiorno nella città emiliana di Paolo III sulla via di Nizza tra il I6 aprile e il 3 maggio I538, evocato nell'opera attraverso un personaggio che si ha esitazione a identificare con l'archiatra pontificio Ferdinando Balami (o Balamio) per solo il fatto che egli sarebbe ricordato di nuovo poco dopo nel testo in maniera più criptica: «mastro Ferrante, buomo valoroso, et dotto [...] quando la corte di sua beatitudine era in Pia- 
cenza, mi esshortò et persuase, che ad ogni modo glielo mandassi) (il formaggio, ossia la Formaggiata stessa). ${ }^{49}$

Nella chiusa del breve scritto Molza è presentato nel Gotha dei burleschi romani della prima e della seconda levatura, accanto a Claudio Tolomei, Giovanfrancesco Bini, Giovanni Della Casa, Caro e a un autore di incerta identificazione: «et veramente era impresa per quei divini, et nella virtù baroni, et principi, et di vostra Maestà servitori affettionatissimi, messer Claudio, il Molza, messer Bino, che si bene ha scritto del suo horto le belle lodi, o di messer Giovanni Della Casa, o di quello che fece il naso dell' anconitano famoso, o quello che cosi elegantemente scrisse della frugalitate»..$^{5 \circ}$ L'ultimo della lista dovrebbe essere appunto il testé ricordato medico di Paolo III, menzionato da Caro nella Statua della Foia con l'epiteto di frugi (Balamio Frugi), ${ }^{5 \mathrm{I}}$ del quale resta un commento al De ossibus di Galeno, che autorizza a pensare si sia esercitato anche sull'opera dietetica del medico greco, il ben noto Della proprietà dei cibi.

Il cenno a Caro tramite perifrasi come celebratore del naso sesquipedale dell'anconitano Giovan Francesco Leoni nella Nasea, ${ }^{52}$ costituisce un ulteriore indizio cronologico. Entrambi $i$ testi risalgono ai primi mesi del 1538 e andarono a stampa insieme per Antonio Blado alla fine dell'agosto I539, il Commento, dopo una rapida e incontrollata diffusione manoscritta, che sorprese l'autore. Nominare la Nasea al posto del Commento come opera più rappresentativa di Caro dopo questa data sarebbe stato impossibile, perciò è gioco forza concludere non solo che la Formaggiata precede la stampa bladiana del Commento con la Nasea, ${ }^{53}$ ma anche che Landi quando compose il suo scritto non aveva nozione del Commento. Il suo scritto va considerato come il contributo di un letterato esterno, che si esercitò con ricettiva tempestività sulle nuove che gli giungevano da Roma per via epistolare, poiché non risulta un suo soggiorno in questo periodo nella città (ma sappiamo poco de suoi spostamenti). Perciò non è inverosimile che le sue informazioni fossero incomplete ed egli fosse al corrente dell'attività dei Virtuosi e quindi dello scritto cariano destinato espressamente ai rituali dell'Accademia, mentre gli sfuggisse l'esistenza del piu importante Commento, che, pur ritraendo il medesimo ambiente, si presentava come un essai autonomo.

Cambia la prospettiva l'esplicito richiamo al Commento di Ser Agresto nella nota Ai lettori della Formaggiata, a firma dello 
stampatore Grassino, che impone di leggere l'avantesto dell'elogio formaggesco dalla specola della lettera del Barbagrigia premessa alla stampa romana del I539: "quel dottissimo gentilhomo et da bene, il Molza sotto 'I nome di padre Siceo, si è sfogato per un tratto, et tuffatosi tutto in fino al gomito ne le lodi de le dolce et saporite fiche, frutto degnamente da lui cotanto lodato e [...] messer Anibale Caro, spirito gentile et capriccioso, ha coperto et adumbrato il suo capriccio sotto 'l nome di sere Agresto». ${ }^{54}$ Si introduce cosi una divaricazione tra il testo della Formaggiata e la premessa, che sposta quest ultima a ridosso della stampa, aprendo qualche interrogativo sulla quiescenza cui lo scritto fu destinato nel lasso intercorso tra composizione ed esito tipografico, senza toccare la questione dell'autoralità della premessa, per la quale sembrano meno pesanti le credenziali a favore di Doni rispetto a quelle dello stesso Landi.

Anche della prosa burlesca di Iacopo Bonfadio intitolata Delle lodi della furfanteria al furfante re della furfantissima furfanteria eletto discute in queste stesse pagine Enrico Garavelli, che inclina a spostare lo scritto dal milieu romano - frequentato da Bonfadio dello scorcio degli anni Trenta e nel quale lo aveva posto l'editore moderno - ${ }^{5}$ a non prima della fine del I543 e lontano dall'Urbe, in forza di una tardiva lettura da parte di Bonfadio della Statua della Foia di Caro. L'eterogeneità dell'operetta di Bonfadio rispetto alle prose della Virtù si palesa in maniera abbastanza nitida. Lo stile compilativo che satura la scrittura riporta la Furfanteria al genere degli elogia paradossali, sul modello del De incertitudine et vanitate scientiarum di Cornelio Agrippa di Nettesheim e dei Paradossi di Ortensio Lando, ovvero di esercizi meno limpidi, ma non per questo meno apprezzati dai contemporanei, come quelli di alcune epistole di Niccolò Franco, ma che tutti non hanno a che fare con l'erudizione sobria e noncurante che emerge dagli scritti dei Virtuosi. Con tutto cio, l'ambientazione dell'operetta di Bonfadio, che si finge composta a Roma, si rivela meno posticcia di quanto si potrebbe pensare. Quando si viene a parlare dei travestimenti cui ricorse furfantescamente Giove per sedurre le donne di cui si era invaghito, ci si imbatte in una interessante memoria di Molza: "Cosi quando volse calcar Leda, si vesti da pollaruolo. Et per questa causa dice il nostro Molza, che di quella pregnezza ella partori due nova». ${ }^{56}$ Il riferimento è all'elegia Ova tibi alpinis nivibus certantia Thyrsis, in cui Molza sotto il 
nome pastorale di Tirsi manda in dono un canestro di nova ad Alessandro Farnese (Alessi). L'elogio della loro candidezza e della perfetta forma oblunga si chiude con l'invito ad accoglierle sulla mensa; nova cosi perfette sono pari a quelle dalle quali vennero alla luce Castore e Polluce, se si deve credere alle favole mitologiche: «Et merito: in vanos ficta est vel fabula lusus / vel nasci his Laedae pignora debuerant). Inedito, come la maggior parte dei carmi molziani, il carme fu incluso dall'autore nella tarda silloge autografa delle sue Elegiae (II IO), ora ms. Vat. Borgiano lat. $367 .{ }^{57}$

Dopo l'exploit del I538 è probabile che la Virtù non rinnovasse il successo della prima stagione e già l'anno dopo non si riuscisse a rinnovare le riunioni con cadenza regolare. In questa vacanza si inseri la sperimentazione avviata da Claudio Tolomei intorno al progetto di poesia barbara, che coinvolse nuovi personaggi e si concluse con la stampa per Antonio Blado, verso la fine del I539, dei Versi e regole della nuova poesia toscana. É probabilmente dopo la conclusione di questa esperienza che Claudio Tolomei si sforzò di mantenere in vita l'Accademia secondo le consuetudini d'un tempo, coinvolgendo $i$ soci anche per il canale epistolare. Il 20 maggio Is40 da Forli Caro si giustificava infatti con Giovan Francesco Leoni per non avere inviato alla Virtù $i$ componimenti promessi. I saluti con cui si chiude la lettera lasciano intravedere una conventicola ricostituita, con Molza partecipe in posizione, al solito, eminente: «raccomandatemi a tutti i padri virtuosi, e sopra tutti al padre Molza». ${ }^{58}$ Su impulso di Tolomei e con l'innesto di nuovi personaggi la Virtù si indirizzò tuttavia verso un programma più nettamente orientato all'indagine antiquaria, che forse per un certo tempo convisse con l'indirizzo burlesco delle origini, per poi prevalere in maniera esclusiva. Ebbe cosi luogo una nuova Accademia, che si denominò Vitruviana e intraprese lo studio del De architectura, riunendo dotti in varie discipline, necessarie all'interpretazione del complesso testo dell'autore latino. Il disegno dettagliato di «questo nuovo studio d'architetturà è consegnato a una lettera di Tolomei ad Agostino Landi, che gliene aveva fatto richiesta, il I4 novembre I542 e descrive un progetto - con la prudenza con cui si devono prendere le date dell'epistolario di Tolomei - allora agli esordi. ${ }^{59}$

Un'ulteriore evoluzione sarebbe l'Accademia dello Sdegno, nelle cui fila confluirono molti dei Vitruviani, con l'intenzione di proseguire il progetto originario rimasto incompiuto, ma le date e le modalità di 
questi passaggi sono ancora in larga parte oscure ed è difficile precisare il ruolo svolto dai vari protagonisti. ${ }^{60}$ Significativo è anzi che l'evoluzione di tali sodalizi, con modifiche delle intitolazioni e degli organici, sia avvenuta senza gravi momenti di stasi, a riprova di una attività culturale non intermessa, che si piegò con duttilità a indirizzi nuovi e che dietro $i$ segnali di discontinuità con $i$ precedenti impegni celava l'evolversi di una esperienza proseguita senza fratture laceranti. Il che si deve anche rovesciare nel giudizio di incapacità, da parte dell'intellettualità romana in questa congiuntura, di partorire un'impresa culturale solida e condivisa, nella quale convogliare in maniera durevole l'attività dei tanti studiosi di varia estrazione e provenienza gravitanti nell'Urbe, con ricadute importanti sul piano della visibilità e dell'identità collettiva, che era stato invece il tratto saliente dell'esperienza dell'Accademia Romana tra il volgere del XV e il principiare del XVI secolo. Non è altresi un caso che le esperienze maturate in seno ai Virtuosi volgessero a un epilogo tutto sommato repentino, approdando a un sostanziale insuccesso nella loro ambizione di scoprire nuove vie percorribili alla letteratura volgare, al di fuori della canonizzazione classicistica che cominciava a prendere piede. Ciò vale per il Commento cariano, audace sperimentazione intorno a una ipotesi di comico colto, che non aveva precedenti ravvisabili nella tradizione volgare, in prevalenza toscana, da cui pure pescava largamente, né si poteva appiattire sulla premessa costituita dall'autocommento berniano alla Primiera. Altrettanto dicasi per la sperimentazione avviata da Claudio Tolomei concretizzatasi nei Versi e regole della nuova poesia toscana, che muoveva dall'esigenza avvertita di sradicare l'esercizio poetico in volgare dal solco di un petrarchismo di cui si cominciava a intuire la deriva essoterica, per attingere a forme più ricercate ed elitarie. ${ }^{6 \text { I }}$

Neppure è sorprendente che da questi incroci inediti tra retaggi classici e terreno del volgare, eseguiti da letterati quasi tutti ferratissimi sul versante latino e greco, si trascorra al terreno per molti rispetti più stabile e accogliente dell' antiquaria, che inibiva ibridazioni del genere di quelle avviate da Caro e Tolomei, ed era in grado di raccogliere in un progetto ecumenico personalità di diversa provenienza e dai curricula non per forza uniformi, unite dalla devozione alla classicità nella città che ne era stata la culla e ne conservava le vestigia. Poiché, tuttavia, ricostruire la parte che ebbe Molza in questa vicenda esula dall'orizzonte di questo saggio, possiamo tornare alla materia burlesca. 
Nella cronologia che si è ricostruita fin qui della presenza di Molza nelle accademie burlesche romane dei decenni Trenta-Quaranta fa la sua irruzione un testo eteroclito. Nel Is 40 a Parma apparve un libretto in ottavo di I6 carte recante la seguente intitolatura e note tipografiche:

CCapitulo in lode del | Verno: Et vno altro | Capitulo i[n] lode | dela Torta | de .M. | Francesco Molza op[er]a | dignisima $\boldsymbol{z}$ no[n] piu vista $\mid$ al $\mathrm{p}[\mathrm{re}]$ sente stanpata ad $\mid \mathrm{i}[\mathrm{n}]$ stantia de Hyp|polito deto | Ferrare|se.| .M.D.XXXX.

col.: Stampata in Parma p[er] fra[n] cesco da prato. $^{62}$

Il frontespizio presenta una cornice xilografica con cordoncini intrecciati su sfondo bianco, nella quale sono compresi sui lati lunghi, due perparte alternati, quattro medaglioni con teste maschili, due clipeate e due togate, rappresentanti le prime condottieri, le seconde, verosimilmente, oratori o magistrati. La modesta qualità della cornice e la sua estraneità al contenuto del libretto, unite al taglio piuttosto arcaico dei caratteri gotici, configurano una stampa a carattere popolare di fattura attardata. Con maggiore pertinenza la cornice è impiegata in un altro opuscolo fatto imprimere da Ippolito a Parma nello stesso anno, questa volta però sottoscritto da Antonio Viotti, il Lume di Marte ocorrente al arte militare, di cui si può solo dire che è posteriore all'elezione di Paolo III (ottobre I534), che vi è nominato.

Ippolito Ferrarese (o da Ferrara) è interessante figura di cerretano ed editore di testi poetici volgari, attivo in diverse città dell'Italia settentrionale e centrale nel quarto e quinto decennio del XVI secolo. ${ }^{63} \mathrm{Di}$ Francesco da Prato si conosce solo un'altra edizione, la Expositio orationis Dominicae a sacrae Theologiae professore Magistro Antonio ex oppido Mathelicae oriundo de agro Piceno, in quarto di 58 carte, recante il suo nome nella sottoscrizione: "Impressum Parmae per Franciscum de Prato Anno Domini M.D.xxxv. Mense Octobris». ${ }^{64}$ Poichè tutte e tre le stampe furono impresse con la stessa serie di caratteri, la conclusione più logica è che essa fosse di proprietà di Antonio Viotti, unico stampatore attivo sulla piazza di Parma in quegli anni, fino al subentro nel I545 di Seth Viotti, già operante come libraio. ${ }^{65}$ Si ha però qualche remora a vedere in Francesco una figura di editore o libraio, che diede il suo nome a due stampe isolate, fatte imprimere nell'unica officina esistente. ${ }^{66} \mathrm{Ne}$ risulterebbe 
una moltiplicazione delle figure coinvolte nelle sue stampe che si ba difficoltà a giustificare per un'opera di infima levatura come la miscellanea poetica del I540, per la quale si avrebbe un committente (Ippolito), un editore (Francesco), uno stampatore (Viotti). Per la stampa del I535, superiore per impegno e risultato, il ruolo subalterno di Francesco è confermato dal fatto che fu l'autore a finanziare la stampa e si procurò il privilegio perpetuo del governatore, il vescovo di Segni Lorenzo Grana, a noi già noto altrimenti (Parma era dominio pontificio dal I5I2). Sulla base di questi dati, nella effimera presenza di Francesco da Prato negli annali tipografici del XVI secolo si deve intravedere piuttosto un operatore occasionale, che stampì per altri in due circostanze isolate e senza rapporto tra loro, forse un dipendente della tipografia Viotti, cui fu concesso di imprimere con il suo nome $i$ due opuscoli su richiesta di personalità esterne all'ambiente cittadino, che per questo usufruirono di un trattamento insolito.

Il frontespizio dell'edizione Is40 non rispecchia il contenuto del libretto, che si rivela più nutrito. Eccone il regesto:

cc. $a_{\mathrm{ii}} r-\mathrm{a}_{\mathrm{iv}} r$. Capitolo in Laude del. Verno $\mid$ Messer compare se vi raccordate

c. $\mathrm{a}_{\mathrm{iv}} r-v$ : Sonete. | Signeri ho tolto una casa a Pisone

c. $a_{\text {iv }} v$ : Sonete del .S. Franchesco molza modenese. | cum un belissimo capitulo in lode de la Torta | Ornar si vede di Terrene Spoglie

cc. $\mathrm{b}_{\mathrm{i}} r-\mathrm{c}_{\mathrm{ii}}$ : Capitolo de la. | Torta $\mid$ Meser Iacomo mio siate pur certo

c. $c_{\text {iii }} r$ Soneto. | Spiazulo Magino: oregia il griselate

cc. $\mathrm{c}_{\mathrm{iii}} \nu \mathrm{d}_{\mathrm{ii}} v$ : Lamento di Roma uno Capitolo della $\mid$ morte del Signor Gioanni de Medici | Hai sconsolata me misera Roma

cc. $\mathrm{d}_{\mathrm{iii}} r_{\text {-iv }} r$ Capitolo sopra la morte del Signor Gio|anni de Medeci | Poscia che in stil si altiero et bellice arte c. $\mathrm{d}_{\mathrm{iv}}$ : : bianca.

Il Capitolo in laude del verno è opera del rimatore friulano Quinto Gherardo ed è a stampa nelle sue Terze rime piacevoli del I537, senza sottoscrizione del tipografo, ma Venezia per Agostino Bindoni, e nelle sue Rime, pubblicata con data di Roma $I^{\circ}$ agosto 1538 , di nuovo senza indicazione dello stampatore, ma, vi si legge, per l'iniziativa di Francesco Amadi, il medesimo responsabile della stampa a Venezia delle Rime di Antonio Brocardo e di altri, cioè anche di Molźa e di Niccolò Dolfin, in cui videro la luce un cospicuo lotto di poesie molziane 
senza l'autorizzazione dell'autore. Il capitolo gherardiano è trasmesso inoltre, adespoto, dal codice di Philadelphia, University of Pennsylvania, Rare Book and Manuscript Library, 4I2, cc. I47v-IsIr, di cui meglio più avanti. ${ }^{67}$

Una cautela liminare va posta sull'edizione sedicente romana, che continueremo a considerare tale nel discorso che segue, sebbene su di essa si debba far pendere il dubbio d'ufficio si tratti di un luogo di stampa fittizio, in attesa che un esame dei caratteri acclari il punto. Cosi come assai dubbio è che responsabile di essa sia Amadi, sul quale non si hanno notizie di attività fuori dal contesto veneziano ${ }^{68}$. Per il capitolo l'edizione parmense riproduce il testo della princeps veneziana, discostandosene solo nella fonetica e per un certo numero di errori. L'edizione romana presenta invece un testo divergente in maniera sostanziale e migliore, per il quale si deve pensare a una redazione d'autore più avanzata. Lo stesso vale per Signeri [sic] ho tolto una casa a pisone, che è il sonetto quarto delle Rime gherardiane, indirizzato lì a un non identificato "Signor Alessandro de Lupria", tipico componimento in stile bernesco incentrato sulla descrizione di una squallida dimora. ${ }^{69}$ La redazione Roma I538 presenta una coda di una sola terzina, mentre in quella Parma I540 sono due, e anche il resto, senza considerare le corruttele della squalificata edizione parmense, mostra differenze consistenti. Il testo I540 è più legato alla dimensione veneziana (a parte il settentrionalismo del primo verso, al $v .3$ troviamo il venetismo carampane $e$ al $v$. I9 gheto) e portatore di un certa esuberanza del dettato, che viene asciugata nella redazione 1538 , più concentrata ed efficace, sulla quale pure doveva avere agito la lima dell'autore. Poiché il sonetto di Amadi All'autore collocato in principio delle Rime, dopo quello Ai lettori, prospetta per l'edizione romana una chiave di lettura esplicitamente antiaretiniana, se ne ricava che la rottura di cui si ba notizi i nei rapporti tra il poeta friulano e il Divino di A rezzo intervenne dopo la stampa veneziana dei Capitoli nel I537 e l'edizione sedicente romana fu un espediente per aggirare l'ostracismo che l'ostilità di Aretino era verosimilmente costata a Gherardo nell' ambiente delle tipografie veneziane. Nel sonetto proemiale Amadi esordisce cosi:

Io v'ho fatto stampar questo Libretto, messer Quinto polito alto et galante, acciò che più non dica ogni furfante che voi non sete tra' 'poeti eletto; 
e nelle due terzine la scelta di collocare Gherardo sulla linea dei burleschi romani emerge come dato saliente dell'impresa editoriale promossa nell'Urbe:

Il Bernia, il Mauro, messer Gianni, et Bino non pur sol imitate, ma passate, tal che di stizza creppa l'Aretino;

il qual, in tutta quanta la sua etate non ha potuto far un Sonettino che dia piacer leggendo a le brigate.

Alla luce di questi squallidi versi l'intera edizione romana di Gherardo reclama dunque un'attenzione che non ha ancora ricevuto.

I capitoli Ahi sconsolata me misera Roma e quello sopra la morte di Giovanni de' Medici della stampa parmense sono opera dell'udinese Eustachio Celebrino, diffusi a partire dal Is 28 insieme con il poemetto in ottave dello stesso Celebrino La presa di Roma (in cui Ahi sconsolata me misera Roma è rifuso). ${ }^{70}$ Il sonetto adespoto Spiazulo Magino, oregia il griselate è scritto in furbesco o in qualche altro zergo di area veneta, come inducono a ritenere alcuni tratti fonetici (il passaggio da c palatale a $\mathrm{z}$ sorda, la prevalenza di consonanti scempie, le forme vintiun $e$ Michiel). Il significato si rivela però un autentico rebus, probabilmente anche per colpa del compositore, che non dovette capire neppure lui nulla del testo e lo guastò in vari punti. Dubito che abbia un senso ciò che si legge al v. 6: «per far sa pollo dai mu[n] portar la palma», dove probabilmente si deve recuperre un "Apollo» e un «Aimun»; mentre al v. I4 «starai calcado in la spagna da iolfo» sarà da leggere in fine «d'Aiolfo», cioè Aiolfo del Barbicone, protagonista di un cantare cavalleresco trecentesco.

Dei due componimenti attribuiti a Molza è escluso che possa essere riconosciuto al raffinato poeta modenese il modestissimo sonetto su una certa Florida, con il testo compromesso da diversi guasti che ne ostacolano la comprensione. Lo si dà di seguito con alcune proposte di restauro:

Ornar si vede di terrene spoglie, di nuovi fior la bassa nostra sphera zephyro, poi che or la prima vera rinveste gli arbosei di verde foglie; 
la pianta amendolina i fior racoglie, poi ch'è passata la rigida e fiera aspreza de l'inverno, cossì altiera che arida stava alhor senza le foglie.

Cossì quel che di fior al nome elletto fiorir si vede ognor de gentileza, de virtù, leggiadria et honestade,

Florida dico, al cui divin aspetto, Florida el sangue et Ferrara in alteza ridopia che altra dona de sua etade.

8. senza le foglie] al nome eletto 9. elleto ı. fiorire

La pianta amendolina al $v$. s sarà il mandorlo, che fiorisce ai primi tepori della primavera; al $v$. I3 una ripetizione del tipo di quella occorsa al $\nu$. 8 rende incomprensibile la seconda terzina.

Di altra fattura si presenta il Capitolo in lode della torta, scritto in un toscano privo di solecismi e di buona levatura stilistica, che mostra $i$ caratteri tipici del capitolo bernesco di lode. Il componimento è databile con sicurezza a dopo il 25 settembre I534, data di morte di Clemente VII (c. $\left.c_{i i} r\right)$; meno utile ai fini della cronologia di quanto sia importante per stabilire la cultura dell'autore è la menzione della Cazzaria di Marcantonio Vignali $\left(c . b_{i v} v\right)$, le cui edizioni si avvicendarono tra il Is30 e il I540.7

Composta da un poeta non sprovvisto di letture di rango, la Torta presenta una generica affinità con $i$ capitoli molziani di sicura attribuzione, in quanto l'una e gli altri sfruttano $i$ passaggi obbligati del repertorio bernesco: invenzione moderna dell'oggetto lodato, polemica con la cosiddetta età dell'oro in cui esso era ignoto, descrizione della preparazione del manufatto e suo impiego. Peculiare della Torta è invece il largo spazio concesso alla polemica antifratesca, che non ba luogo nei capitoli molziani. Al contrario, la Torta si può dire abbia la sua principale intenzione nel prendere in giro gli uomini di Chiesa, siano essi frati, prelati o papi (Clemente VII e l'altro papa mediceo Leone X), tutti universalmente accomunati dal vizio della pederastia. Né l'autore ha timore di attingere nel suo anticlericalismo toni blasfemi, come quando prende in prestito l'elogio del fegatello che Margutte fa in Morgante, ХVIII I26, aggiungendo un'appendice di irriverente parodia, della quale avverte la gravità: 
Margute vuol che la fusse tra sante la prima e l'hebbe in gran devotione, come è scripto sul libro di Morgante, et féli un Credo et una confessione, et ogni dì com un torchieto in mano devoto la dicea in genochione.

Ma questo sente un po' del lutherano, et se Margute a dì nostri el dicesse, gli saria fatto un scherzo molto strano. ${ }^{72}$

Troppo per un poeta fine e ironico come Molza, che anche quando nei suoi ternari attinge alla satira anticlericale, lo fa con un certa arguzia briosa, lontano dalle movenze grevi del poeta della Torta, senza considerare che con $i$ suoi 324 versi essa supera di parecchio le misure dei tre capitoli moliziani, oscillanti tra gli appena I2I della Scomunica $e$ i circa 220 dell'Insalata $e$ dei Fichi. Altra dissonanza è la rinuncia dichiarata da parte del poeta della Torta alla spiegazione dell'etimologia, che invece è sfruttata da Molza nel Capitolo della insalata (vv. 76-84). Da ultimo, in generale, si considererà l'inconvenienza di un capitolo che esalta la sodomia per un poeta amante del gentil sesso come Molza, il quale dedica due dei suoi capitoli, quello Della insalata $e$ quello Dei fichi, alla lode dell'organo femminile e quando nella Scomunica decide di celebrare il rapporto contro natura lo fa senza ricorrere a una metaforica pesante come quella impiegata dall'autore della Torta.

Respinta fino a prova contraria la paternità molziana del capitolo, si ripropone in tutta la sua pertinenza la domanda su come mai l'attivissimo e girovagante Ippolito Ferrarese sia responsabile della messa in circolazione di due apocrifi sotto il nome di un poeta decisamente illustre e nella sede ancora periferica di Parma anteriore all'avvento del ducato farnesiano, eppure singolarmente ricettiva, se si considera che ad Antonio Viotti si deve, nello stesso I538, la princeps non autorizzata, $e$ non sottoscritta, delle Rime di Vittoria Colonna. Sia il capitolo sia il sonetto pseudomolziani non hanno una posizione di particolare eminenza nel libretto, rivelando una strategia un po' insensata se si intendeva dare risalto all'opera di un poeta celebre rispetto ai componimenti adespoti raccolti nel volume.

L'edizioncina parmense arrivava due anni dopo la stampa piratesca di un cospicuo lotto di sonetti e canzoni molziane, delle ottave sul ritratto 
di Giulia Gonzaga e della Ninfa Tiberina, nelle ricordate Rime del Brocardo et d'altri authori. Se Ippolito avesse avuto in mente di spendere in maniera eclatante il nome di Molza anche alla luce di quell'episodio, avrebbe organizzato l'edizione con altri criteri. Esclusa l'ipotesi di una consapevole contraffazione, si deve concludere che abbia dato ai torchi $i$ due componimenti cosi come gli erano giunti tra le mani, senza porsi troppi interrogativi sul fatto che uno dei due recasse in epigrafe un nome importante, che non si poteva più dire sconosciuto al pubblico del libro a stampa. Non si ha perciò difficoltà a rubricare l'episodio sotto l'etichetta di un sottoprodotto dell'industria tipografica dell'epoca, interessata a smerciare operette di poco o nessuno rilievo letterario, in una sede nella quale mancava l'attenzione di un pubblico ampio e consapevole, che potesse svolgere un'azione di inibizione e di controllo rispetto a iniziative squalificate come quella di cui stiamo parlando. Se Molza fu avvisato per tempo della prossima uscita della stampa Amadi a Venezia e tentò invano di bloccarla in tutti $i$ modi, per l'edizioncina parmense si può dare per certo che non ne sapesse nulla.

Il Capitolo in lode della torta ebbe una discreta circolazione manoscritta. Girolamo Tiraboschi nella Biblioteca modenese afferma che a Modena ne esistevano diverse copie..$^{73}$ Una di queste con il titolo La torta di M. Francesco Molsa si trova in un duerno scritto da mano cinquecentesca legato nel codice Estense $W 2$ II (lat. 228), cc. $238 r$-24Ir $(E)$, monumentale silloge di carte sciolte e fascicoli eterogenei per provenienza e aspetto, riuniti nel XVII secolo e rilegati nell'attuale codice all'epoca in cui Tiraboschi era bibliotecario dell'Estense (1780-94). ${ }^{74}$ Altro esemplare databile al XVI secolo è il Magl. VII $\operatorname{II}_{8}(M)$, dove il capitolo In lode della torta si trova adespoto alle cc. CXIIr-CXXv, all'interno di una silloge di numerose rime e qualche prosa, per lo più adespote, trascritta da una sola mano, che diede unità all'insieme numerando $i$ componimenti per metro (il nostro è il sesto dei capitoli). Ancora al XVI secolo risale il ms. Landau Finaly 136 della Nazionale centrale di Firenze (LF), scritto da diverse mani, di cui una prevalente, contenente una raccolta omogenea di oltre I40 capitoli burleschi adespoti, ma preceduta da una tavola degli autori scritta dalla mano principale. Il codice contiene il Capitolo della insalata con il nome di Molza (cc. 29Ir-294v) e quello Della torta senza nome dell'autore (cc. $416 v-422 v)$. 
Il testimone più importante della Torta è però il Palatino 269 della Nazionale di Firenze (P), che contiene l'edizione delle liriche molziane allestita dal pronipote del poeta, Camillo, e pronta per la stampa, come attesta la nota Ai lettori e la dedica al principe Cesare d'Este in data Is aprile I6I4, stampa che poi non fu eseguita per motivi che restano ancora ignoti. Il nostro capitolo fa la sua comparsa alle cc. 257r-265v, quasi in fondo alla seconda delle tre parti in cui sono divise le rime, quella contenente le poesie inedite, dopo le edite della prima parte e prima dei poemetti della terza (una quarta parte conteneva le novelle, ma fu separata dal codice tra il 1747 e il I 889 ed è ora il ms. Casanatense 3890 ). Il fatto che, evidentemente, la stampa parmense del capitolo sfuggi all'attenzione di Camillo non desta stupore, poiché l'esame della raccolta mostra che il censimento delle ben note antologie di rime cinquecentesche a stampa fu condotto in maniera incompleta e confusa, perciò nulla di piu facile che Camillo non avesse notizia della stampa del I 540 , di certopoco nota già al principio del XVII secolo, e ricorresse a un manoscritto.

L'editore settecentesco delle rime di Molza Pierantonio Serassi, venuto in possesso degli inediti di $P$, allora di proprietà dell'erudito napoletano Francesco Valletta, nella premessa del primo volume dell'edizione accenna al capitolo, «non mai accennato ch'io sappia da veruno Scrittore», ${ }^{75}$ ma un esame più attento lo decise ad accantonarlo, come argomentò diffusamente nella premessa al secondo volume: "Debbo poi avvertire, che il Capitolo in lode della Torta, che fu detto trovarsi tra le Rime inedite del Molza, dopo d'averlo io attentamente esaminato, e fattolo ancora esaminare da varie letterate Persone, si è giudicato non essere assolutamente opera del nostro Poeta, non solo per lo stile, e maniera di verseggiare assai bassa, e per $i$ motti scipiti e freddi dissimili affatto da que' degli altri graziosi ed eleganti Capitoli del Molza; ma eziandio per molt altre sconvenevolezze, che vi si veggono perentro e che lo dimostran Opera di qualch'altro Poeta vicino al diciassettesimo secolo». ${ }^{75}$ Probabile che a decidere Serassi per l'espunzione fosse soprattutto la sconvenevolezza citata per ultima, piuttosto che lo stile basso $e$ $i$ motti scipiti. Ma la conclusione circa la data del componimento mostra come l'erudito settecentesco non avesse una chiara visione storiografica della nostra letteratura, a giudicare possibile che un poeta comico scherzasse sulla religione citando Lutero in Italia in età postridentina. Del resto, gli scrupoli morali di Serassi investirono anche i tre capitoli autentici, giudicati piacevolissimi nella vita di Molza nel I volume, 
ma «ne' quali non si può desiderar cosa alcuna che all'ultima perfezion si appartenga, fuorché in alcuni alquanto più di modestia», ${ }^{77}$ al punto che - e cio desta stupore - solo quello Della insalata fu accolto nell'edizione, tratto dalle Opere burlesche, Giunti I548, e vennero lasciati fuori la imbarazzante Scomunica, ma anche il più famoso dei tre, quello Dei fichi.

Nella seconda metà del Cinquecento la fama del Capitolo della torta con attribuzione a Molza non fu effimera. Tommaso Garzoni nel discorso CI della Piazza universale di tutti i mestieri del mondo, del I585, che tratta De' maestri delle scienze e costumi, e de' putti che vanno a scuola, lo portò ad esempio di testo osceno che nonostante $i$ divieti circolava tra gli scolari: «non si tiene gran conto di tener la Pippa, la Nanna, le figure aretinesche, la torta del Molza, il Bernia, il Burchiello, il Franco, idolo de' studenti, benché si facciano tante inibizioni al contrarios. ${ }^{78}$

La considerazione che Garzoni aveva della Torta è confermata dalla sua presenza nell'elenco di elogi paradossali proposti in apertura de Il mirabile cornucopia consolatorio, breve esercizio in questo genere retorico dedicato da Garzoni alla celebrazione delle corna: "Benché al primo incontro la materia da trattarsi paia disdicevole e vile, - esordisce la consolatio garzoniana - nondimeno, a quella guisa che Diocle dal celebrar la rapa, Xanto l'ortica, Protagora il ravanello, Virgilio il pulice, il Calcagnino la cimice, il Molza la torta, e simili altri scrittori gravi diverse altre bassezze magnificando s'apersero la strada a una magnifica e onorata fama...». ${ }^{79}$ L'elenco va preso con prudenza: Virgilio cantò il culex, la zanzara; il pulice, la pulce, è materia di un famoso elogio di Celio Calcagnini; degli elogia agresti dei tre autori greci che aprono la serie, il primo è una dotta estrapolazione della Naturalis historia di Plinio (XX I9), gli altri non so. Infine, sospetto legittimo èche la Torta sia finita nella lista, unico esemplare in volgare e solo moderno accanto a quello umanistico di Calcagnini, per un effetto di sciame provocato dal Culex virgiliano, che avrebbe richiamato un altro componimento dell'Appendix, il Moretum. Come che sia, resta da constatare che nel ragionamento di Garzoni l'onore e l'onere di fare da anello di congiunzione tra tradizione classica e umanistica delparadosso ed elogio in stile bernesco spetta a questo testo di tradizione incerta, che non attecchi negli annali tipografici della letteratura burlesca, né ha sollecitato finora l'attenzione della critica. 
A completare il nutrito dossier tortesco del XVI secolo occorre ricordare $i$ capitoli del palermitano Gian Guglielmo Bonincontro (Sovviemmi della vostra rima accorta), del modenese Giovan Francesco Ferrari (Haveste il torto a fé, messer Roberto), ${ }^{80}$ del senese Pietro Nelli (Malipiero, hoggi io fo quel che io non soglio) $)^{8 \mathrm{I}}$ e il sonetto del perugino Francesco Beccuti (Non cerchiam più se Ligurino è sordo). ${ }^{82}$ Solo il primo stabilisce un rapporto con la Torta pseudomolziana, che Bonincontro menziona espressamente. Rivolgendosi a Gerardo Spada, governatore di Monreale e membro dell'Accademia palermitana degli Accesi, cui anche Bonincontro fu affiliato, egli dichiara:

E a dire incominciai spinto da vui più lodi della torta che non disse il Molza già, ch'io ne so più di lui. ${ }^{83}$

Sulla biografia e sull'opera di Bonincontro esiste qualche incertezza, poiché fu a lungo confuso con il fratello Mariano, probabilmente per responsabilità di Giovan Battista Giraldi Cinzio, che nel Discorso sul comporre dei romanzi riporta un aneddoto di Gian Guglielmo con il nome di Mariano, scambiandolo con il fratello, dato che entrambi avevano studiato diritto a Ferrara a pochi anni di distanza. ${ }^{84}$ Nato verso il I520, Gian Guglielmo soggiornò a Genova dal I535, prima di recarsi per studio a Ferrara, dove si addottorò in utroque il 9 luglio I545. Dunque, nulla di più facile che, se coltivava la poesia di indirizzo burlesco, fosse venuto a conoscenza della stampa parmense del I540. In una data imprecisabile tra il 1548 e il I5 8 (Giraldi, che scrive prima del I553, parla di lui come se non fosse già più a Ferrara) fece ritorno a Palermo, dove ricopri un pubblico ufficio, ma incorse nei rigori dell'Inquisizione per avere professato idee luterane e subi il carcere. Il Capitolo della torta fu composto durante la prigionia, probabilmente tra il settembre e l'ottobre I567. E' uno sterminato capitolo autobiografico di Iso terzine, in cui Bonincontro, mostrando non comune forza d'animo, ripercorre i momenti felici della sua vita e sfoga l'amarez?a per la disgrazia presente, consolandosi al pensiero della torta di cui ha fatto in passato solenni scorpacciate e prendendosi una solenne rivincita con i potenti del mondo intero (compresi alcuni ordini religiosi), dipinti tutti come grandi consumatori di torte. 
I tre capitoli autentici di Molza, Dei fichi, Della insalata $e$ Della scomunica, sono disponibili nell'edizione curata in anni recenti da Mirella Masiero, ${ }^{85}$ corredata da copiose note, cui è stata rimproverata $^{86}$ una adesione supina alla formula interpretativa elaborata da Jean Toscan nell'ormai classico Carneval du langage. ${ }^{87}$ Nella Nota ai testi Masiero dichiara di essersi attenuta per $i$ capitoli Della insalata $e$ Della scomunica all'editio princeps, cioè alla citata stampa veneziana delle Terze rime del Berna et del Mauro per Curzio Navò del Is39 (N); per il Capitolo dei fichi, invece, ha preferito il Libro secondo delle Opere burlesche edite dai Giunti di Firenze tra il 1548 e il I55s ( $F$ ) alla princeps nel Commento di ser Agresto da Ficaruolo di Annibal Caro impressa a Roma da Antonio Blado nel I539. Una tavola propone le varianti delle edizioni delle Opere burlesche del XVIII secolo. A parte incidenti dovuti a una trascrizione delle stampe adottate non sempre impeccabile e alla messa a testo di mende che avrebbero richiesto un intervento risanatore, il limite oggettivo dell'edizione è di non avere affrontato l'esame della tradizione manoscritta, che, dal punto di vista della critica del testo, obbligherebbe per uno dei capitoli a rifondare il lavoro su altre basi. E quanto si intende avviare qui di seguito, illustrando $i$ risultati di una prima recensio sui codici.

La tradizione dei capitoli di Molza non è unitaria. Quella manoscritta dà risultati molto diversi, ma anche quella a stampa, cui si è appena accennato, dopo le principes distinte di Insalata $e$ Scomunica da un parte e Fichi dall'altra, si unifica solo a partire dalla vulgata, l'edizione delle Opere burlesche pubblicate da Firenze da Bernardo Giunti e dagli eredi, nella quale però, non a caso, $i$ primi due capitoli figurano insieme nel Libro primo (I548), mentre $i$ Fichi trovano posto nel Secondo (I555) e comunque continuano ad essere diffusi insieme con il Commento di Caro in una tradizione distinta. Solo dell'Insalata resta un numero ragguardevole di manoscritti, che consentono di ricostruire una storia della trasmissione; per gli altri due le testimonianza manoscritte sono rare o inesistenti e ci si deve attenere alle stampe. Perciò ci si concentrerà in questa sede sulla Insalata, limitandosi per gli altri due capitoli ad alcune annotazioni.

Della Scomunica non sono emersi manoscritti; solo la prima terzina isolata con la corretta intestazione "Capitolo della scomunica del 
Molza» si legge a c. 44r del Magl. VII 372, del XVI secolo, raccolta di poesie bernesche che si riprometteva di essere di grandi dimensioni se il codice conta numerose carte bianche rigate fino alla fine, ma che aborti proprio con l'incipit del capitolo molziano. ${ }^{88}$ Viene da chiedersi se l'impatto con l'argomento toccato sia stato cosi traumatico da inibire la prosecuzione del lavoro di copia. Il Capitolo della scomunica è dedicato all'uso di affiggere in luoghi pubblici e frequentati cartelli famosi o ritratti satirici con $i$ quali mettere al pubblico ludibrio personaggi noti e solo collateralmente tocca la scomunica come condanna canonica. ${ }^{89}$ L'elogio tessuto da Molza di questo costume diffuso nella città di Pasquino, ma non solo, insiste nel rovesciare l'endoxon secondo il quale ciascuno preferisce si parli bene di se stesso, anziché male, e nel presentare come gradevole e vantaggiosa la diffamazione, che per interpretatio viene a significare la sodomia passiva, solo apparentemente fastidiosa e invece apprezzabile. L'argomento era più scabroso dell'elogio dell'amore eterosessuale insito nell'Insalata e nei Fichi, ma ciò non costituirebbe - nel panorama burlesco del secolo, fitto di presenze analoghe - un intralcio bastevole alla sfortuna del capitolo rispetto agli altri due. ${ }^{90}$ Si deve pensare piuttosto al significato canonico del termine prevalso in epoca tridentina e al contenuto diffusamente anticlericale del capitolo. Fattori, questi, sufficienti a far sparire la Scomunica dalla linea tipografica dopo le Opere burlesche impresse a Venezia da Domenico Giglio nel is64-66 $(G)$, che conserva $i$ tre capitoli molziani con la stessa distribuzione di F tra Primo e Secondo libro e senza infierire sul testo.

La collazione di $N$ e F non dà risultati di rilievo. Il testo è pressoché identico, salvo pochi interventi di $F$, che non sono di ostacolo a ipotizare un ascendente comune se non addirittura $F$ in posizione descritta. Una censura è al v. 22, mentre al verso successivo $F$ equivoca un termine neutro e lo sostituisce con uno adeguato al contesto:

$\mathrm{N}$

22-23.

Dio sa che volentier anch'io'l farei

ma perchéaddosso un nembo me ne viene
F

Dico che

membro 
Alv. 8 F Femenda unguasto:

$\mathrm{N}$

Dipingemi chi vuol con scura faccia

ma aivv. go e go interviene senza necessità:

$\mathrm{N}$

88-90.

O beneficio grande a chi ben mira

non essere fastidito da persona

che ti faccia a sentirla angoscia od ira

99 .

senza voltar di clementina o sesto
F

Dipingami; esser

che ti faccia sentir l'angoscia

[ol'ira

Si tratta, in quest'ultimo caso, del gesto di volgere le carte dei ponderosi tomi del Corpus iuris canonici, dove sono pubblicate le raccolte di decretali di Clemente Ve il Sesto libro aggiunto da Bonifacio VIII ai cinque precedenti di Gregorio XI.

Il Capitolo dei fichi è trasmesso con il nome di Molza dal codice di Philadelphia, University of Pennsylvania, Rare Book and Manuscript Library, 4I2, cc. 56r-62bis recto $(P h) .{ }^{9 \mathrm{I}}$ Il codice consiste in una raccolta di 32 capitoli burleschi del XVI secolo per la maggior parte sprovvisti dei nomi degli autori, benché quasi tutti noti: alle cc. I06v-IIzv ospita anche, adespoto, il Capitolo dell'insalata. I Fichi, come pure l'Insalata, sono presenti anche nell'edizione delle liriche molziane eseguita dal pronipote del poeta Camillo Molza trasmessa nel Palatino 260 della Nazionale di Firenze con la data Is aprile I6I4 (P). ${ }^{92}$

Per $i$ Fichi la collazione di $F$ con $P h$ dà come risultato la vicinanza dei due testimoni, ma la dipendenza di Ph da F è da scartarsi a priori per il semplice fatto che il responsabile di $\mathrm{Ph}$ mostra di non conoscere l'autore dell'Insalata. A parte questa isolata attestazione manoscritta, l'edizione del Capitolo dei fichi risulta legata a quella del Commento di Caro, per il quale oltre alla stampa bladiana del I539 sono emersi frammenti di una tradizione manoscritta, che, se importante in primo luogo per la storia interna del Commento, risulta indispensabile anche ai fini della restituzione del testo del capitolo. ${ }^{93}$ Nell'impossibilità di affrontare la questione in questa sede, mi dedico al capitolo 
molziano per cui la recensio ba dato esiti più incoraggianti $\mathrm{Del}$ Capitolo della insalata sono emersi tre manoscritti. Oltre a $\mathrm{Ph}$, dove è presente, adespoto, alle cc. I06v-IIzv, l'Insalata si legge invece con il nome dell'autore nel già ricordato Landau Finaly I36, cc. 29Ir-294V $(L F)$, nel quale si trova anche il capitolo Della torta. ${ }^{94} \mathrm{Nel}$ Vat. lat. $8262(\mathrm{~V})$, voluminosa silloge fattizia di versi e prose, in massima parte lettere, del XVI e XVII secolo, il capitolo occupa le cc. II-I4, con l'intestazione originaria "Del Molza», cui è stato aggiunto dopo "Dell'insalata». Si tratta di quattro fogli sciolti scritti integralmente su entrambe le facciate, incollati su strisce di carta per consentirne la legatura nel codice. Il foglio I4 è legato per errore in ultima posizione mentre è il secondo della serie (tracce di usura sono presenti a c. I3v). I fogli furono sottoposti a piegatura in quattro, verosimilmente per inserirli in una missiva, sebbene la presenza di un rinvio in calce a c. I2v sembrerebbe indicare la fine di un fascicolo e perciò l'unità codicologica originaria potrebbe non essere un duerno, bensi le carte finali e iniziali di due fascicoli contigui. Di mano posteriore è l'intervento censorio al $v$. I2: " "per lei diventi se'l dicesse Dio» > "per lei diventi anco al dispetto mio». Infine, come si e anticipato, l'Insalata fu inclusa nella sua edizione da Camillo Molza.

Sul versante delle stampe, la recensio che segue tiene conto, naturalmente, di $\mathrm{N}$ e F, ma prende in considerazione anche il Libro primo delle Opere burlesche impresse a Venezia da Domenico Giglio nel I564 $(G)$, le Rime piacevoli del Berni, Casa, Mauro, Varchi, Dolce, et d'altri Auttori edite a Vicenza per Barezzo Barezzi nel I603 $\left(V^{\top}\right)^{\prime}$ e la ristampa di Francesco Grossi nel I600 $\left(V^{2}\right)$, sebbene tutte descritte da F e le ultime due espurgate, ma necessarie per stabilire la posizione di P. Tralascio, le ristampe giuntine del I550, I552, I555, e la ristampa di Vc, Venezia, Francesco Baba, I627. ${ }^{95}$

Per ragioni di spazio si lasciano fuori anche le edizioni settecentesche delle Opere burlesche, e cioè: Il primo libro delle opere burlesche di M. Francesco Berni, di M. Gio. Della Casa, del Varchi, del Mauro, del Bino, del Molza, del Dolce e del Firenzuola, Londra, Per Giovanni Pikard, I72I (edizione curata sotto lo pseudonimo di Antinoo Rullo da Paolo Rolli, con le note di Antinoo Nivalsi, cioè Anton Maria Salvini); Il primo libro dell'opere burlesche. Di M. Francesco Berni, di M. Gio. della Casa, dell'Aretino, de' Bronzini, del Franzesi, di Lorenzo de' Medici, del Gali- 
leo, del Ruspoli, del Bertini, del Firenzuola, del Lasca, del Pazzi, e di altri autori, In Firenze [Napoli, G.M. Porcelli] I723; Il primo libro dell'opere burlesche di M. Francesco Berni, del Molza, di M. Bino, di M. Lodov. Martelli, di Mattia Francesi, dell'Aretino, et di diversi Autori, In Usecht al Reno [Roma], Appresso Jacopo Broedelet, I726. Per opinione corrente tali edizioni sono considerate ininfluenti per la restitutio textus, invece alcuni saggi hanno smentito la loro passività rispetto alle stampe giuntine del XVI secolo e talora si sono svelate portatrici di buone lezioni. Resta da acclarare se esse siano frutto di emendamenti ope ingenii o di ricognizione sui codici.

I rapporti fra $i$ testimoni sopra elencati si compendiano graficamente nel seguente stemma codicum:

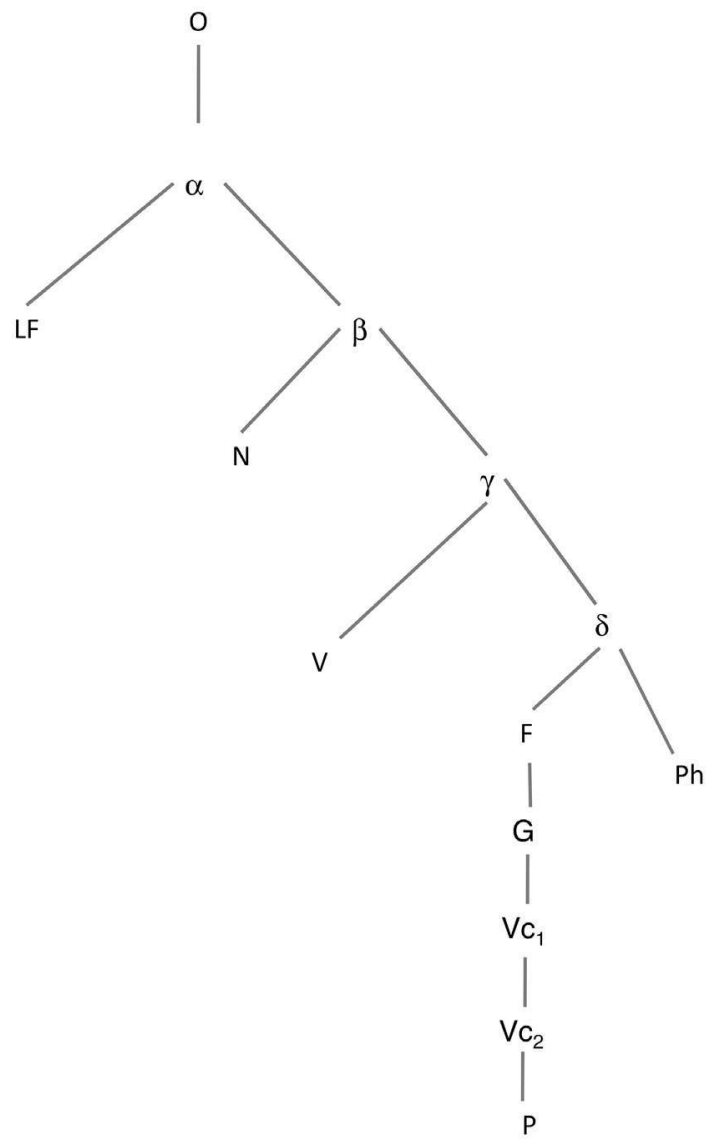


Assai facile l'eliminazione del ramo veneto costituito da $G$ e dai suoi sottoposti. La dipendenza di $V c^{I}$ da $G$ e non da $F$ si dimostra, oltre che con la generale aderenza di $V C^{I}$ alla grafia di $G$, in particolare con:

I 2.

$\mathrm{F}$

$\mathrm{G}, \mathrm{Vc}^{\mathrm{I}}$

per lei diventi se 'l dicesse Dio

Pio

26.

da far strebiliar chi su vi pensa

starbiliar.

$V c^{I}$ è, come è noto, edizione pesantemente censurata e, oltre a eliminare la Scomunica, sottopone Insalata $e$ Fichi, mantenutisi integri fino a $G$, a un'energica potatura di tutto quanto concerne la religione e delle oscenità troppo esplicite. L'Insalata risulta cosi mutilato dei vv. 49-54, con maldestri interventi di sutura nei lembi, e modifiche meno traumatiche interessano ivv. 20, 42, 84, I13, I48, I68, 194, 21I, 217. Nei Fichi sono manomessi per ragioni di fede $i$ versi 46, II9-I20, I42, I63, I77, I8I-I83 (con perdita della catena rimica), 217-219 (con taglio di tre versi); per morale sessuale $i$ versi 65, 85-87, I00, II0, II5, I34-136 (interventi meno caratterizzati riguardano ivv. II7, I33). In più, la modifica ai vv. 28-30 rivela scrupoli di natura politica:

ceteri

$\mathrm{Vc}^{\mathrm{I}}$

Altro fregio fu questo e viè più bello

di quel che'l doge di Vinegia adorna di quel che'lgran Bassà si cinge adorno allor ch'al Bucentoro apre il portello. quando esce fuor del stretto Dardanello.

La dipendenza di $P$ da $V c^{2}$ è provata dal seguente errore:

v. 145

ceteri $\mathrm{Vc}^{2}, \mathrm{P}$

non mi vi spiace et hor che 'l caldo è grande piace.

Due gli errori archetipici presenti in tutta la tradizione (eccetto $V c$, $P$ mutili in questo punto). Dove l'invenzione dellinsalata viene fatta risalire miticamente al primo nomo ovvero al santo eremita Ilarione (alludendo con perfidia alla dieta vegetale degli anacoreti?), si constata la mancanza di monumenti che celebrino l'uno o l'altro, con questa chiosa: 
52-54.

Sia pur come si voglia ha sì gran merto, a sì gran o fusse Adamo il primo o Ilarione, poco era un tempio ad ogni statua certo od ogni.

I seguenti errori di LF hanno valore separativo da $\beta$ :

$\mathrm{LF}$

I 42 - I 44 .

quanto meglio i Lombardi che 'l gentile fanno meglio suo cascio parmegiano o piacentino

v'aggiugono con più saggio e chiaro stile I 45-I 47 .

Qualche fior leggiadretto et peregrino non mi vi spiace, et hor che il caldo è grande un citruolo affettato ho per divino I 53 .

d'ogni viver che fussi almo ed egregio I96.

O sopra gli altri illustre condimento

2II-2I3.

Chi t'ama esser non può se non beato

e chi la mente tiene a te rivolta

vivrà con poca spesa in ogni lato $\beta$

(1)

\author{
$\operatorname{affettarvi}(\mathrm{N}) ; \operatorname{affettar}(\gamma)$ \\ che s'usi \\ ogni altro (ogn'altro $\mathrm{Vc}^{\mathrm{I}}, \mathrm{Vc}^{2}$, \\ P)
}

vive (vivea $\mathrm{N})$.

Al fraintendimento della lezione originale, forse su un testo già guasto o poco chiaro dell'antigrafo, piuttosto che a un intervento deliberato, si deve con ogni probabilità la lezione di LF al v. 64, che comporta la modifica della sintassi dell'intera frase rispetto alla versione trasmessa dal resto dei testimoni (turbata in $N$ ):

\section{$\mathrm{LF}$}

6I-64. non di men ne la lingua delli hebrei ne la latina ne la greca antica ne quella forse ancor de gli Aramei voce non truovo onde el suo suon [ne dica $\beta$

non dimen né la lingua delli hebrei néla latina né la greca antica né quella forse ancor de gli Aramei voce ritrova (ritorna $\mathrm{N}$ ) onde 'l [suo nome dica. 
$F, P, P h, V, N(\beta)$ sono a loro volta uniti contro $L F$ da un errore:

$\beta$

I60-162.

Io per me volontier mai non l'assaggio

se sotto sopra non la meno io stesso

e vi meno più volte e di vantaggio rimeno;

valore congiuntivo va conferito anche a due diffrazioni:

$\mathrm{LF}$

88-90.

A che risponder brevemente io voglio

e conchiudendo tosto la sentenza

lassarmi a drieto ancor quest'altro scoglio lassarvi lasciar lassarai

(ove la lezione corretta trasmessa da LF si era guastata a monte degli altri testimoni, con tentativi da parte di ciascuno di dare una risposta migliorativa, a parte $F$, che altera in malo modo);

LF

$\mathrm{N}$

$\mathrm{F}, \mathrm{Ph}, \mathrm{V}$

I93-I95.

Questo è quel che di lei più ch'altro

loda

ogni buon monsignor, ogni convento

che nelle cene lor s'uson per coda che de le certe lor l'usan perché certi di lor per coda

l'usan percoda

(la lezione di LF è l'unica che dà senso in quanto si riferisce all'uso di consumare verdure a fine pasto; lascia incerti il passaggio dal singolare al plurale dal I al III verso della terzina, riferiti sempre all'insalata - di lei, s'uson -, che suggerisce di recuperare l usan da $\beta$ : «che nelle cene lor l'usan per coda»).

La famiglia $\gamma(F, P h, V)$ è distinta dai seguenti errori:

$\gamma$

108-109.

entrar di migliorarla in ferma spene

né volse come pria mangiarla asciutto
$\mathrm{N}$

entrò 
I 48 - I 50.

Spesse (Molte $\mathrm{Ph}$ ) volte io disprezzo (sprezzo $\mathrm{Ph}$ ) le vivande in disprezzo quanto vuoi delicate e di gran pregio mi son cadute, come fosser ghiande I 45 -I 47 .

Qualche fior leggiadretto et peregrino non mi vi spiace, et hor che il caldo è grande un citruolo affettar ho per divino

affettarvi è fregio

L'insalata non mai perch'ella ha 'l fregio d'ogni ben ricca mensa anzi è la luce i65-i66.

non mi venga chi m'ama a porsi appresso

ch'io faccio le pazzie e tutto il mondo faria.

Si aggiungano varianti di $\gamma$ rispetto al testo concorde di $L F, N$ :

$\gamma$

76-78.

Echisa che'l suo nome entro (entra $\mathrm{Ph}$ ) la torre

di Babele non restasse (restosse $\mathrm{Ph}$ ) impastoiato

elà si stia poi ch'altri nol soccorre

I04.

da chi si fosse il trovator da bene

I 84 .

forse (forsi V) che doppo lei non dà buon bere? ${ }^{96}$

I 87 .

l'insalata o'l popone, e chi s'intende

209.

il nome ceda anchor ceda l'agliato

cui si unisce una lezione caratteristica:

I 29.

ma darvi dentro baldanzoso e ardito chi chi

LF, N

Echiha

impanïato

vero è che doppo lei non dà buon

[bere

melone

et ceda anchor;

Particolarmente marcata è la sostituzione lessicale al v. I87, che identifica senza possibilità d'errore il responsabile di $\gamma$ come toscano. "Io stetti già per creder che 'l popone» principia il Capitolo del bacio di Della Casa ed è nota la veemenza con cui Grazzini difese il vocabolo 
fiorentino da quello forestiero (che a Firenze designa un insipido tipo di zucca) nel suo autocommento al Capitolo in lode della salsiccia, probabilmente in polemica proprio con il fortunato capitolo Dei fichi di Molza, reso celebre dal commento di Caro, il quale capitolo esordiva appunto con "Di lodare il mellone avea pensato». Scrive Grazzini:

con i forestieri la voglio, con quelli solamente che fanno professione della nostra lingua, quei primi, quei maggiori i quali son degni veramente di immortal gloria, e li domando a che fare o con quale autorità si muovano a chiamare i poponi melloni, con ciò sia cosa che l'una la miglior frutta sia e la più saporita, e l'altra la più trista e scioccha che si mangi, perciò che il mellone tiene il mezzo tra il cetriuolo e la zuccha, e da ciascuno de' due piglia il più cattivo. Una frutta certo che non ha né odor né sapore, però non si trova se non qualche bambino, qualche svogliato, o qualche donna gravida che ne mangi, mal fatta, sproportionata quanto la può e sopra tutto sciocchissima. ${ }^{97}$

Poiché $N$ presenta a sua volta errori dai quali $\gamma$ è immune, si deve postulare che $\gamma$ non sia descritto da $N$ ed entrambi discendano da $\beta$ :

\section{$\mathrm{N}$}

37.

Però quando vi avien che ciò contempli

64.

voce ritorna onde el suo suon ne dica I 27-I 3 I.

Nè bisogna toccarla con un dito come alcun sciocco, ch'imbratarsi teme ma darli dentro baldanzoso e ardito, empirsene le man, la bocca insieme, senza ch'altri t'inviti se n'ha brama I 8 I .

Per un quatrin tanta abondanza v'hai I93.

Questo è quel che di lei ch'altro loda 2I I-2I3.

Chi t'ama esser non può se non beato e chi la mente tiene a te rivolta vivea con poca spesa in ogni lato

\section{$\gamma$}

mi avien (mi avvien $\mathrm{V}$, m'avvien $\mathrm{Ph}$ )

ritrova

hai

n'hai.

più ch'altro. 
All'interno di $\gamma$ una duplice lezione particolare e un errore separano $\delta$ $(F, P h) d a V:$

$\delta$

I, 3 .

Un poeta valente mi promesse

l'ingegno altrove poi e l'opra messe IOI.

perché dico che 'l nascer l'insalata
V

promise

mise

$V$, a sua volta, è separato da $\delta$ da una lezione da considerarsi erronea:

$\mathrm{V}$

I 34 .

ove menar si possa con destrezza $\delta$

prestezza.

La lezione archetipica, difficiliore e più perspicua, è prestezza. Si discute infatti qui dell'agevolezza nella penetrazione della vagina («il tondo largo di ragion sempre ama» v. I33) rispetto all'ano ("Sempre mi parve di color sciocchezza / che le fan con l'aceto sol la festa» vv. I36-I37) e prestezza indica la rapidità e facilità con cui si svolge l'atto, mentre destrezza esprime piuttosto perizia, cautela o particolare abilità richiesta dal rapporto sodomitico, cioè il contrario di quanto Molza vuole comunicare nel suo gioioso panegirico del sesso secondo natura.

In effetti, $V$ ha tra $i$ testimoni un profilo un po' particolare, non solo per le caratteristiche materiali della trasmissione, ma anche per un certo numero di errori banali che lo caratterizzano:

$\mathrm{V}$

6. s'ei sottometteva le spalle a cotai some

27. ed io poco atto a ragionar vi sono

30. isporre ad altri la sua bontà immensa

6r. nondimeno néla lingua de gli Hebrei

I o s. e così l'insalata fu nominata

I 8 . fin qui troppo alto habbiamo disteso il

[volo

I 54. Talhora la sera a casa si conduce ceteri

sommettea (somettea $\mathrm{N}$ )

ne sono

bontà sua

non di men (nondimen $\mathrm{Ph}$ )

nomata

habbiam (habbian LF)

talhor (LF, N) tal'hor (F, Ph) 
I64. per pescar meco nel medesimo tondo 206. a me basta sperar in te corona medesmo

basti.

La frequenza con cui viene adottata la forma piena di un sostantivo o di un aggettivo con l'effetto di produrre ipermetria è prova di una certa rigidità nell'ortografia, che fa pensare a uno scrivente che avesse dimestichezza con la penna (come denota anche la scrittura, regolare ma con tratti tachigrafici come la o quasi sempre aperta e la forte riduzione di alcune lettere in legamento, ad es. $\mathrm{t}, \mathrm{r}, \mathrm{s})$, ma non necessariamente familiarità con il verso, piuttosto attenzione meccanica alla correttezza grammaticale che finezza d'orecchio, forse un segretario, o un cancelliere, o qualche ufficio consimile. Altri fenomeni, che non incidono sulla ortopedia, come la forma piena del dittongo al posto di quella ridotta per caduta della semivocale (tipo ai per a') o della preposizione articolata con raddoppiamento fonosintattico al posto della forma discreta (tipo della per de la), percepita probabilmente come arcaica, rientrano nel profilo di uno scrivente abituato a conferire al testo la veste grammaticale e ortografica ritenuta corretta e percio, in linea di principio, disponibile a un certo grado di manipolazione.

$F, P h$ presentano tra loro errori separativi che escludono l'uno sia descritto dall'altro. Ph si caratterizza per un maggior grado di trascuratezza:

$\mathrm{Ph}$

65.

questo s'io non m'inganno un gran diffetto

questo è 66.

u' quanto altrui più pensa più s'intrica

altri 89.

e conchiudo tosto la sentenza

conchiudendo io6-107.

ma puoi ch'a lungo andar come interviene ma poi a lungo andar.

Una variante adiafora completa il panorama:

$\mathrm{Ph}$

F

I 48 .

molte volte io sprezzo le vivande

spesse volte. 
Errore di $F$ è il seguente:

$\mathrm{F}$

$\mathrm{Ph}$

88-90.

A che risponder brevemente io voglio

e conchiudendo tosto la sentenza

lassarai a drieto ancor quest'altro scoglio

lasciar.

Illustrata la ricostruzione stemmatica che si proposta in principio, resta da affrontare il comportamento di alcuni testimoni che in apparenza la contraddicono e richiedono un supplemento di analisi. LF, e V condividono una lezione corretta a dispetto del resto della discendenza di $\beta$ :

I6-I 8.

LF, V

$\mathrm{N}, \delta$

ch'a mastro Febo non vo' dar affanno

e men turbar le Muse, ch'in disparte

a goder l'ombre del suo monte

tuo.

L'errore si spiega con l'invocazione al v. Iz: «Aiutami, Trifon, tu, ch'a la meta), che ha attratto nella sua orbita l'aggettivo possessivo e la lezione corretta si potrebbe spiegare con un emendamento autonomo di LF, V, entrambi capaci di buone intuizioni. Lo stesso dicasi per una lezione adiafora comune a LF, $V$, che potrebbe nascere da un riflesso grammaticale altrettanto compatibile con il profilo dei due codici, inclini a intervenire sulla sintassi:
LF, V
$\mathrm{N}, \delta$

I 68.

se mi chiamasse il papa non rispondo se mi chiamasse (chiamassi $N$ ) il papa ('l papa $\mathrm{Ph}$ ) io non rispondo.

Che lo stemma codicum proposto non esaurisca sic et simpliciter le problematiche di una tradizione che si è rivelata complessa accade per un paio di lezioni. Al v. I2 Ph e G, con tutti $i$ suoi subalterni, restituiscono contro $F$ la medesima lezione sbrigativamente censurata:

$\mathrm{Ph}, \mathrm{G}$

per lei diventi, se 'l dicesse Pio
$\mathrm{F}$

dicesse Dio. 
Si ricordi che anche in V una mano posteriore era intervenuto sul passo con un più elegante "per lei diventi anco al dispetto mio», percio si potrebbe anche qui invocare per Ph, G una poligenesi, per una sorta di automatismo scattato alla prima vistosa menzione di cose sacre e rimasto senza seguito, dato che nessuno dei due testimoni si distingue per intenti censori, che invece saranno preoccupazione dominante di $V c^{I}$. Al v. 96 interviene però un caso analogo e vieppiù interessante che coinvolge anche $V$ :
$\mathrm{Ph}, \mathrm{V}, \mathrm{G}$
F, LF, N
dovea parer una casoccia un forno
casaccia.

Osteggia un poco quanto scrive Varchi nell'Hercolano a proposito dei derivati di casa: «Similemente quando alle parole di genere masculino s'aggingne otto, o vero occio, e a quelle di femminino otta, o vero occia, si cresce il lor significato, come casotto, casotta $e$ casoccias. ${ }^{98}$ Nell'Insalata si tratta piuttosto di un'abitazione angusta e fatiscente, anche oscura e fetida, come suggerisce di rincalzo forno, ma ai derivati, come ambito espressivo della lingua, non si può applicare una semantica rigida ed è legittimo recuperare il vocabolo difficiliore con un senso genericamente avvilitivo. Sul piano stemmatico, mentre è facile spiegare con una banalizzazione poligenetica la presenza di casaccia in LF, N, è invece problematico comprendere come mai dia la stessa lezione anche $F$, dove ci si aspetterebbe casoccia, risalente fino a $\gamma$.

Non credo che anche in quest'ultimo caso ci siano gli estremi per invocare la contaminatio. Resta spazio per una congettura più interessante, cioè che sia esistito uno stato differente di F portatore della lezione archetipica casoccia presente in $\gamma$ e da un esemplare recante questo stato sia stata ricavato $G$, mentre la copia da me consultata (Biblioteca apostolica Vaticana) dovette essere impressa dopo che sulle forme erano state introdotta la lectio facilior casaccia. La verifica sugli altri esemplari di $F$ conservatisi potrà dare forse una risposta a questa che al momento non è che una ipotesi: non è molto confortante che EDITI6 ne censisca solo cinque.

Franco Pignatti 
I. Anton Francesco Doni, La libraria, a cura di V. Bramanti, Milano, Longanesi, i 972, pp. 4io-i i; Anton Francesco Doni, I mondi e gli inferni, a cura di P. Pellizzari, Torino, Einaudi, I994, pp. 20-2 I; cfr. S. Longhi, Lusus, Padova, Antenore, I 982, pp. 43-44 n.; D. Romei, Da Leone X a Clemente VII. Scrittori toscani nella Roma dei papati medicei (I513-1534), Manziana, Vecchiarelli, 2007, pp. 205-207 (I ed. Firenze, Centro 2P, 1984).

2. Francesco Saverio Quadrio, Della storia e della ragione d'ogni poesia, I, Bologna, F. Pisarri, I739, p. 96: ai nomi della lettera di Sabino Quadrio aggiunge però anche quello di Molza; Girolamo Tiraboschi, Storia della letteratura italiana, VII, I, Venezia, I796, pp. I33-34; anche SAverio Bettinelli, Delle lettere e delle arti mantovane, Mantova, Er. A. Pazzoni, I774, p. I 30 . Poi M. Maylender, Storia delle Accademie d'Italia, V, Bologna, L. Cappelli, I930, pp. 466-67.

3. Le terze rime del Berna et del Mauro e I capitoli del Mauro et del Bernia et altri authori, entrambi Venezia, C.T. Navò, I 537; Capitoli del Mauro, del Bernia, del Varchi et d'altri eccellenti poeti, Roma, s.t., I 539 .

4. Cfr. la voce Giova (Jova), Giuseppe, di Stefano TAвасchi, in Dizionario biografico degli Italiani, LV, Roma, Istituto della Enciclopedia italiana, 2000, pp. 427-29.

5. È la conclusione cui giunge F. BAIOcchi, Sulle poesie latine di Francesco Maria Molza, in «Annali della Scuola Normale di Pisa», XVIII (I905), p. 79.

6. Doni, I mondi e gli inferni, p. 23; per le altre opere citate si veda la nota di Patrizia Pellizzari a p. 2 I e Romei, Da Leone X a Clemente VII, cit., pp. 207-208.

7. «Concluderemo, dunque, negando credibilità documentaria alle indicazioni doniane e avvertendo che, se vorremo conservare all'accademia il nome dei "Vignaiuoli", d'uso ormai consolidato, dovremo essere consapevoli che si tratta di un'etichetta di comodo, convenzionale ed inesatta»: cosí Romei, Da Leone Xa Clemente VII, cit., p. 209. Si cita per dovere la biografia di Molza di Pierantonio Serassi, la quale, con grave pregiudizio della cronologia, colloca i Vignaiuoli al I 538 (Delle poesie volgari e latine di Francesco Maria Molza corrette, illustrate, ed accresciute colla vita dell' Autore scritta da Pierantonio Serassi, Bergamo, P. Lancellotti, I 747-54, I, p. LI).

8. Francesco Berni, Poesie e prose, a cura di E. Chiorboli, Genève-Firenze, L.S. Olschki, I934, p. 35 i.

9. L. D’Ascia, Erasmo e l'Umanesimo romano, Firenze, Olschki, I991, pp. i 8 5-87.

ı. Per inciso, le concordanze delle rime berniane allestite da Romei nel sito www.nuovorinascimento.org permettono di constatare che divino è impiegato una sola volta con intento antifrastico, a proposito della mula di Guazzalletto medico nell'ipercaudato sonetto a lui diretto $O$ spirito bizzarro del Pistoia, al v. 45. Con normale estensione semantica per indicare qualità eccelse trovo divino/divina in un luogo delle lettere (ed. cit., p. 33 I).

i i. Berni, Poesie e prose, p. 357, p. 364, p. 366 ; cfr. A. Corsaro, La regola e la licenza. Studi sulla poesia satirica e burlesca fra Cinque e Seicento, Manziana, Vecchiarelli, I999, pp. 73-74. 
I 2. Lo stesso si può dire della sobria chiusa della lettera a Bini del 27 dicembre I 533: «Non altro: a voi et a tutta l'academia mi raccomando» (Berni, Poesie e prose, p. 345).

i 3. Berni, Poesie e prose, p. 363.

i4. Francesco Berni, Rime, a cura di D. Romei, Milano, Mursia, i 985 , p. i 85.

i 5. Benedetto Varchi, L'Hercolano, a cura di A. Sorella, II, Pescara, Libreria dell'Università Editrice, I995, p. 902 (aggiungo una virgola dopo corte, come richiede il senso); una testimonianza analoga sui «modi di parlare» tipici di Molza, cari a Giulia Gonzaga, nella lettera di Annibal Caro a Molza da Napoli del i 8 maggio i 538 (Annibal Caro, Lettere familiari, a cura di A. Greco, I, Firenze, F. Le Monnier, I957, p. 97 e anche p. 79) e anche una lettera di Giacomo Cenci ad Alessandro Corvini del 5 marzo i 45 (De le lettere facete, et piacevoli di diversi grandi nomini, et chiari ingegni, raccolte per M. Dionigi Atanagi, Libro primo, Venezia, B. Zaltieri, I 565 , p. 277).

I6. Delle poesie volgari e latine di Francesco Maria Molza, II, I750, p. 86, modifico l'interpunzione.

I7. BERNi, Rime, pp. I73-74.

I8. Corrispondenza Giovanni Della Casa, Carlo Gualteruzzi (I525-I549), a cura di O. Moroni, Città del Vaticano, Biblioteca apostolica Vaticana, I986, p. 9.

19. Relego in nota notizia de La Caccia d'Amore del Bernia, con la risposta del Molza. Et una Giostra da Cavallieri erranti. Con un Capitulo di Pietro Aretino contra le Donne, Venzia, M. Pagan, I 554 (poi Ferrara, V. Panizza, I 562), nella quale almeno le presenze di Berni e Molza sono certamente apocrife.

20. Romei, Da Leone X a Clemente VII, cit., p. 2 I 7.

2 I. Annibal Caro, Gli Straccioni. La Ficheide. La Nasea e La statua della Foia, Milano, G. Daelli e Comp., I863, p. 94.

22. Dicerie di Annibal Caro e di altri a' Re della Virtù, a cura di B. Gamba, CalvelyHall [Venezia, Alvisopoli], i 821, p. 92.

23. Caro, Gli Straccioni. La Ficheide. La Nasea e La statua della Foia, p. I 3 I.

24. Cfr. la voce di Adriano Prosperi nel Dizionario biografico degli Italiani, VIII, I 966, pp. 203-204, e L. Dorez, La cour de Paul III, I, Paris, Librairie E. Leroux, I932, pp. 37-4I.

25. La possibilità che Trifone sia arrivato a Roma a una data anteriore è legata al celebre sonetto berniano Ser Cecco non può star senza la corte, nel quale è preso in giro suo zio Francesco, segretario alle lettere latine sotto più pontefici, ed egli è nominato nella chiusa: «Ma da poi la sua morte, / arassi almen questa consolazione, / che nel suo loco rimarrà Trifone». Il sonetto è collocato dagli studiosi di Berni tra quelli composti sotto il pontificato di Clemente VII, anteriori al Sacco, 
quindi si risalirebbe di sette-otto anni rispetto al i 534-35, il che anagraficamente è possibile, se si tiene conto che Trifone morì nel $\mathrm{I} 57 \mathrm{I}$, ma in assenza di notizie positive a conferma di tale inferenza è preferibile attenersi alla data più tarda.

26. Il 24 dicembre I 539 da Forlì Caro scrive a Molza: «Non mi curo, ch’ella mi scriva altramente di sua mano; ma di grazia, commetta a messer Trifone che mi faccia talvolta un verso, secondo che andrà avanzando della sanità» (CARO, Lettere familiari, p. I67).

27. T.F. Mayer, Reginald Pole. Prince and Prophet, Cambridge, Cambridge Universiy Press, 2000, p. 64. A questo viaggio allude il Commento di ser Agresto, dove nell'ampio elogio dedicato Trifone si legge «avendo letto, che i Francesi vennero a combattere di qua per le nostre Fiche, egli ha voluto passar di là a combattere per le Fiche di Francia; dove intendo che ha fatto prove stupende, benché ultimamente ci abbi lasciato il pelo» (CAro, Gli Straccioni. La Ficheide. La Nasea e La statua della Foia, p. I 32 ).

28. Benci arrivò a Modena probabilmente dopo il 5 giugno i 543 , perché una breve lettera scherzosa scrittagli da Claudio Tolomei in quella data non reca cenni al Molza, come avviene nelle successive (De le lettere di M. Clandio Tolomei lib. sette. Con una breve dichiarazione in fine di tutto l'ordine de l'ortografia di questa opera, Venezia, G. Giolito, I 547, c. I8 Iv).

29. Luca Contile, Ragionamento sopra la proprietà delle imprese, Pavia, G. Bartoli, I 574, c. 42r: «In Roma similmente fu fondata l'Academia della virtù sotto la magnanima autorità d'Hippolito Medici gran Cardinale, questa impresa fu parimente senza Motto perché fra quei famosi Academici, splendor di questo nostro secolo, fu contrasto in qual foggia si dovesse la virtù dipingere, in quel mezo venne lo stesso generoso Cardinale a morte $\langle\mathrm{e}\rangle$ non si ricercò altro, per che volsero alcuni che la virtù fusse morta per la morte di quel Prelato vero mecenate di vertuosi a' nostri giorni». Ma su un'impresa per l'Accademia, consistente nella parola stessa VIRTV, della quale vengono spiegati i significati attraverso la tecnica esoterica dell'interpretatio nominum, discetta Giovanfrancesco Bini nella Cotognata, omaggio a Claudio Tolomei, terzo re della Virtù, databile al i 538 (Delle lettere facete, et piacevoli, di diversi grandi huomini, et chiari ingegni, scritte sopra diverse materie, raccolte per M. Francesco Turchi, Libro secondo, Venezia, [A. Manuzio], I 575, pp. 270-278 e Dicerie di Annibal Caro e di altri a' Re della Virtù, pp. 59-68).

30. Formaggiata di sere Stentato al Serenissimo Re della Virtude, Stampata in Piasenza per Ser Grassino Formaggiaro M.D.XLII, c. 2v.

3 I. CARO, Lettere familiari, p. 59.

32. CARO, Lettere familiari, pp. 69-70.

33. Caro, Lettere familiari, p. 79, p. 83.

34. Sul Commento cariano si vedano gli studi di E. Garaveldi, Presenze burchiellesche (e altro) nel Comento di Ser Agresto di Annibal Caro, in La fantasia fuor de' confini. Burchiello e dintorni a 550 anni dalla morte (I499-1999). Atti del Convegno (Firenze, 
26 novembre 1999), a cura di M. Zaccarello, Roma, Edizioni di storia e letteratura, 200I, pp. I95-239; «Perché Prisciano non facci ceffo». Ser Agresto commentatore, in Cum notibusse et comentaribusse. L'esegesi parodistica e giocosa del Cinquecento. Seminario di Letteratura italiana Viterbo, 23-24 novembre 200I, a cura di A. Corsaro - P. Procaccioli, Manziana, Vecchiarelli, 2002, pp. 57-77; I pentimenti di Ser Agresto. Terza variazione sul Commento alla Ficheide di Annibal Caro, in «Filologia e critica», XXVIII (2003), pp. I 8 I -208.

35. Opere di Benedetto Varchi, II, Trieste, Lloyd Austiaco, I 859, p. 69rb.

36. VARCHi, L'Hercolano, p. 806.

37. Romei, Da Leone X a Clemente VII, pp. 220-22 I; una commendizia di Annibal Caro per Franzesi allo Strozzi con data io febbraio i 538 in CARo, Lettere familiari, p. 6r.

38. Firenze, Biblioteca nazionale, II VII I 29, p. 52, p. 54.

39. Caro, Gli Straccioni. La Ficheide. La Nasea e La statua della Foia, p. i 84.

40. Il secondo libro dell'opere burlesche, di M. Francesco Berni, del Molza, di M. Bino, di M. Lodovico Martelli, di Mattio Francesi, dell'Aretino, et di diversi autori, In Fiorenza, Apresso li Heredi di Bernardo Giunti, I 55 , c. 72r; Caro, Gli Straccioni. La Ficheide. La Nasea e La statua della Foia, p. I 84.

4I. Il secondo libro dell'opere burlesche, c. $73 \mathrm{r}$.

42. Il secondo libro dell'opere burlesche, c. $75 \mathrm{v}$.

43. Non può essere Giulia Gonzaga, come vuole Silvia Longhi (Poeti del Cinquecento, a cura di G. Gorni - M. Danzi-S. Longhi, Milano-Napoli, Ricciardi, 200 I, p. 970 n.), la quale dal dicembre i 535 risiedeva a Napoli e non aveva più avuto contatti diretti con Molza (salvo contraria prova). Allo stesso modo non possono essere le Stanze di Gandolfo Porrino sul ritratto di Giulia eseguito da Sebastiano del Piombo quelle di cui Franzesi chiede ai vv. Io-I 2 del capitolo: «Messer Gandolfo ha fatto ancora grazia / di mostrarvi le stanze sue divine, / ch'io non potei veder per mia disgrazia?». Le Stanze porriniane, così come quelle più famose di Molza sullo stesso soggetto, risalivano al principio del I533 (CAro, Lettere familiari, p. I I). Bisognerà perciò scegliere tra le Stanz̧e di lontananz̧a e le Stanže in laude di Susanna romana, a disposizione nella tarda stampa delle Rime di Porrino (Venezia, M. Tramezzino, I 55 I).

44. Su di ciò F. Pignatti, Benedetto Varchi e il progetto di edizione delle rime di Francesco Maria Molza, in Varchi e altro Rinascimento. Studi offerti a Vanni Bramanti, a cura di S. Lo Re e F. Tomasi, Manziana, Vecchiarelli, 20 I 3, pp. 8 I-Io9.

45. Firenze, Biblioteca nazionale, II VII I 29, p. 54.

46. Per informazioni su di lui rinvio in queste pagine al saggio di Enrico Garavelli. Capitoli e altre scritture burlesche di Panciatichi si leggono nell'autografo D 3 I 3 della Biblioteca Forteguerriana di Pistoia, raccolta di poesie sue e di altri autori, tra i quali Molza, presente con alcuni sonetti seri e burleschi. 
47. Prudente è da considerare la datazione di Contro il parlar per Vostra Signoria proposta da RomeI, Da Leone $X$ a Clemente VII, p. 22 I, che si attiene al termine estremo all'abbandono definitivo di Roma da parte di Molza nel maggio i 543 . Considerata la situazione sanitaria del poeta molto seria già dal I 542 - nell'estate si sparse addirittura la notizia della sua morte - pare impossibile che una persona ridotta in uno stato compassionevole potesse essere scelta per sermoneggiare facetamente sulla superfluità di certe buone maniere.

48. D. Rhodes, Accertamenti sulla Formaggiata del conte Giulio Landi, in «Bollettino storico piacentino», LXXX (1985), pp. 2 Io-I 3.

49. Formaggiata di sere Stentato, c. I7v [ma $2 \mathrm{Iv}]$; M. BAUCIA, Accertamenti storicoletterari sulla Formaggiata del conte Giulio Landi, in «Bollettino storico piacentino», LXXXI (1986), pp. го4-2 г. Su Balami la voce di Paola Zambelli nel Dizionario biografico degli Italiani, V, I963, pp. 307-308.

\section{Formaggiata di sere Stentato, c. $17 \mathrm{r}$ [ma $2 \mathrm{Ir}]$.}

5 i. Caro, Gli Straccioni. La Ficheide. La Nasea e La statua della Foia, p. i 86. Altro personaggio è il «messer Ferrante» che introduce Caro nel gabinetto di curiosità in cui è conservata la statua oggetto della diceria e che probabilmente coincide il «messer Ferrante» nominato da Caro in una lettera a Giovanni Gaddi del 30 aprile I 538 (CARO, Lettere familiari, p. 84).

52. E superius: «el formaggio piacentino per la sua grandezza sarà Re de gli altri cascii, come il naso di messer Francesco d'Ancona è Re de gli altri nasi» (Formaggiata di sere Stentato, c. 9v).

53. È da rivedere la data di Roma i a aprile i 545 di una lettera di Tolomei a Landi, nella quale si fa menzione della Descriptio insulae Maderae, frutto della rielaborazione degli appunti scritti da Landi durante il viaggio nell'isola probabilmente compiuto nel I 529 , che Tolomei aveva visto per un solo giorno e restituita a Paolo Giovio (l'opera sarebbe stata edita solo nel i 574 a Piacenza), ma si allude in cifra anche alla Formaggiata: «Del formaggio che m'inviate, vi ringrazio assai, ma molto più vi ringraziarò, quando sarà arrivato, e molto più senza dubbio, quando io lo mangiarò, e lo trovarò buono, sì come io spero, venendo da coteste parti, e da voì (De le lettere di M. Claudio Tolomei, c. i i $7 \mathrm{v}$ ).

\section{Formaggiata di sere Stentato, c. 2 r.}

55. Iacopo Bonfadio, Le lettere e una scrittura burlesca, a cura di A. Greco, Roma, Bonacci, 1978 , p. 32, p. 36 e passim, non interviene su questa datazione Paolo Trovato nella recensione-saggio Intorno al testo e alla cronologia delle Lettere di Jacopo Bonfadio, in «Studi e problemi di critica testuale», 20 (1980), pp. 29-60, ora in Jacopo Bonfadio a cinquecento anni dalla nascita. Atti del convegno di Roè Volciano (25 ottobre 2008), a cura di A. Bonomi e S. Zaboni, Comune di Roè Volciano, 2009, pp. 55-66, a pp. $57-58$.

56. Bonfadio, Le lettere e una scrittura burlesca, p. i6r. La lettera è adespota in De le lettere facete, Libro primo, pp. 323-336, da dove traggo la citazione a p. 326; è data a 
Bonfadio dal ms. A I 3 della Biblioteca Ambrosiana, confermato da una epistola dell'autore a Fortunato Martinengo del i 545 (Lettere famigliari di Jacopo Bonfadio da Gazano sulla Riviera di Salò, Brescia 1758, pp. 93-94).

57. Francesco Maria Molza, Elegiae et alia, a cura di M. Scorsone - R. Sodano, Torino, RES, I999, p. 59 .

58. CARO, Lettere familiari, p. I95.

59. De le lettere di M. Claudio Tolomei lib. sette, cc. 8 Ir-85r; edita in Scritti d'arte del Cinquecento, a cura di P. Barocchi, III, Milano-Napoli, I977, pp. 3037-46.

6o. Sull'argomento si veda G. VAGEnHeIm, Appunti per una prosopopea dell'Accademia dello Sdegno a Roma: Pirro Ligorio, Latino Latini, Ottavio Pantagato e altri, in «Studi umanistici piceni», XXVI (2006), pp. 2 I I-26; anche A. Schreurs, Antikenbild uns Kunstanschauungen des neapolitanischen Malers, Architekten und Antiquars Pirro Ligorio (I5I3-1583), Köln, W. König, 2000, passim, e P. Procaccioli, Per Tommaso Spica. Testi e note intorno a un accademico 'Sdegnato' della Roma Farnesiana, in Roma e il papato nel Medioevo. Studi in onore di Massimo Miglio, II, Primi e tardi umanesimi: nomini, immagini, testi, a cura di A. Modigliani, Roma, Edizioni di storia e letteratura, 20 I2, pp. $233-53$.

6r. Recensisco in margine il giudizio di un verseggiatore non sospetto di inclinazioni petrarchevoli come Giovanfrancesco Bini nella Cotognata, diretta a Tolomei, terzo re della Virtù: «Et però la Maestà V. insieme con la sua Virtuosissima corte, ha fatto un gran bene alla Republica a proveder con que' nuovi versi a questi sì fatti disordini. Li quali versi havendo voce di prosa, et signification di versi, hanno così del retorico, come del poetico, et è tanto soave così fatta mescolanza, che qualunque li sa ben fare, con sopportatione di tanti salta in panca, che vanno attorno, si può chiamar meritamente poetissimo tra gli oratori, et oratorissimo tra ' poeti» (Delle lettere facete, Libro primo, p. 272).

62. EDITi6 registra un solo esemplare nella Biblioteca Augusta di Perugia (segn. ANT I N I 23 [I]); un altro transitato sul mercato antiquario ne segnala Giancarlo Petrella nella sua recente monografia «Ad instantia d'Hippolito Ferrarese». Un cantimbanco editore nell'Italia del Cinquecento, in «Paratesto», 8 (20I I), pp. 23-80, a p. 57 n. I. Dell'esemplare perugino Giosuè Carducci si fece eseguire dal bibliotecario della Augusta Adamo Rossi una trascrizione, che si conserva, insieme con gli altri materiali molziani, a Bologna, Casa Carducci, cart. XLIII, fasc. I6, 8.

63. Per un panorama approfondito su Ippolito Ferrarese rinvio senz'altro al citato Petrella, «Ad instantia d'Hippolito Ferrarese», pp. 57-58, pp. 75-76 sulla nostra edizione.

64. Su Antonio da Matelica F. Vecchietti - T. Moro, Bibliotheca Picena o sia notizie istoriche delle opere e degli scrittori piceni, I, Osimo, D. Quercetti, I790, p. I 82; G.G. Sbaraglia, Supplementum et castigatio ad scriptores Trium Ordinum S. Francisci a Waddingo aliisve descriptos, I, Rome, A. Nardecchia, I908, p. 87. Gli sono attribuiti anche un Opus de triplici mundo e Sermones de beatae Mariae Virgins festivitatibus. 
65. G. Drei, I Viotti stampatori e librai parmigiani nei secoli XVI-XVII, in «La Bibliofilia», XXVII (1925), a pp. 218-43, pp. 22 I-26.

66. Così pensa, al contrario, Petrella, «Ad instantia d'Hippolito Ferrarese», cit., p. 58, e, prima, la breve nota dedicata a Francesco da Prato nel Dizionario dei tipografi e degli editori italiani, Il Cinquecento, a cura di M. Menato - E. Sandal G. Zappella, I, Milano, Editrice bibliografica, I 997, p. 456, basata però solo sulla Expositio.

67. Nel Secondo libro dell'opere burlesche, Venezia, D. Giglio, i 564 il Capitolo in laude del verno scivola in mezzo a quelli di Mattio Franzesi, cui però nella tavola viene premessa la didascalia «Di M. Quinto Gherardi». L'attribuzione resta incerta anche nelle edizioni settecentesche.

68. D. Perocco, Un testo quasi sconosciuto della questione della lingua nel Cinquecento: il Dialogo de la lingua di Franceco Amadi, in «Studi e problemi di critica testuale», 26, I983, pp. I 17-50; Ead., Nuove postille e osservazioni di Francesco Amadi, in Studi vari di lingua e letteratura italiana in onore di Giuseppe Velli, I, Milano, Cisalpino, 2000, pp. 383-403.

69. 亡̀ edito anche, con incipit corretto, in Alphabeto sententioso, s.l., a istanza di Ippolito Ferrarese, I 539, c. $\mathrm{B}_{2} \mathrm{v}$; cfr. Petrella, «Ad instantia d'Hippolito Ferrarese», cit., p. 75 .

70. G. Milanesi, Il sacco di Roma del MDXXVII. Narrazioni di contemporanei, Firenze, G. Barbèra, i 856 , p. XLV; il Lamento è edito in Lamenti storici dei secoli $X I V, X V$ e $X V I$, a cura di A. Medin - L. Frati, III, Bologna, Romagnoli, I890, pp. $37 \mathrm{I}-77$.

7 i. Arsiccio Intronato (Marcantonio Vignali), La Cazzaria, a cura di P. Stoppelli, Roma, Edizioni dell'Elefante, I984, pp. I 53-54.

72. C. $\mathrm{b}_{\mathrm{iii}}$.

73. G. Tiraboschi, Biblioteca modenese, III, Modena, Società tipografica, I 783 , p. 238.

74. In calce alla Torta Pellegrino Niccolò Loschi, archivista ducale alla fine del XVIII secolo, lasciò traccia della sua indignazione: «L'Autore fu un bel porco, e la Composizione non d'altro è degna che di fuoco».

75. Delle poesie volgari e latine di Francesco Maria Molza, I, I747, c. * Ior.

76. Delle poesie volgari e latine di Francesco Maria Molza, II, I750, p. 5 r.

77. Delle poesie volgari e latine di Francesco Maria Molza, I, I747, p. LXXXV.

78. Tommaso Garzoni, La piazza universale di tutte le professioni del mondo, a cura di P. Cherchi - B. Collina, II, Torino, Einaudi, I996, pp. i I65-66.

79. Tommaso Garzoni, Opere, a cura di P. Cherchi, Ravenna, Longo, i993, pp. $527-28$. 
80. Giovanfrancesco Ferrari, Rime burlesche, Venezia, Er. M. Sessa, i 570, cc. 7 r-Ior. Segnalo la presenza di quattro capitoli di Ferrari adespoti nel codice Vaticano Capponiano i 5 2, cc. Ioor-1 o6v, in un frammento mutilo al suo interno di una carta. Il frammento Capponiano si rivela importante perché presenta suoni e forme settentrionali, nonché alcuni vocaboli diversi dalla stampa, il che alimenta l'ipotesi, già avanzata, che questa sia il risultato della pesante revisione editoriale eseguita dal curatore Borgaruccio Borgarucci. Perciò il manoscritto si rivela prezioso per il ripristino di una buona lezione e ne dovrà tenere conto il futuro editore critico delle rime di Ferrari. Cfr. F. Brambilla Ageno, Un saggio di furbesco del Cinquecento, in «Studi di filologia italiana», XVII (1959), pp. 22 I-37, e A. Corsaro nelle note dell'edizione da lui curata dei Paradossi di O. Lando (Roma, Edizioni di storia e letteratura, 2000), dove sono segnalati i numerosi plagi di Ferrari a spese dell'opera landiana.

8 . Delle satire alla carlona. Libro secondo. Di Messer Andrea da Bergamo, In Vinegia, Appresso Bernardino, et Filippo Stagnini fratelli, MDXLV, cc. 65r-67v: «Torta, a messer Andrea Malipiero. Sat. XVII».

82. A Crismani, Edizione critica delle Rime di Francesco Coppetta dei Beccuti, 2012, $<$ http://paduaresearch.cab.unipd.it>, p. 274.

83. O. Coppoler Orlando, Un poeta bizzarro del 'soo (Mariano Bonincontro da Palermo), in «Archivio storico siciliano», XXX (I905), pp. I-54: 36, vv. I9-2 I.

84. L'aneddoto merita di essere riportato perché caratterizza con efficacia il personaggio: «Per pigliarsi spasso di simili ingegni, faceva (come sapete) i più belli sonetti del mondo, quanto alle voci et alle rime, i quali non dicevano cosa alcuna et erano senza sentimento; poi gli lasciava uscire sotto nome di qualche valent'uomo, et egli stesso si trapponea tra gli altri et mostrava di volerli far sopra discorsi, dicendo che era maraviglioso il senso loro, là onde induceva ognuno a farvi sopra fantasie et opinioni» (Giovan BatTista Giraldi Cinzio, Discorsi intorno al comporre rivisti dall'autore nell' esemplare ferrarese Cl. I go, a cura di S. Villari, Messina, Centro interdipartimentale di studi umanistici, 2002, pp. 90-9I). Per i problemi relativi alla biografia cfr. C.A. GARUfI, Contributo alla storia dell'Inquisizione in Sicilia nei secc. XVI e XVII, in "Archivio storico siciliano», XL (I9I6), pp. 304-389: 349-37 I, e l'efficace sintesi nella voce di Claudio Mutini in Dizionario biografico degli Italiani, XII, I970, pp. 21 2-2 I 4.

85. Francesco Maria Molza, Capitoli erotici, Galatina, Congedo, i 999.

86. Da Floriana Calitti nella recensione in «La Rassegna della letteratura italiana», CV (200I), I, p. 222.

87. J. Toscan, Le carnaval du langage, Lille, Université de Lille III, i98 I.

88. Inventari dei manoscritti delle biblioteche d'Italia, XIII, Forlì, L. Bordandini, I905०6, pp. 79-80.

89. Sull'argomento si veda G. ORTALLI, «...pingatur in Palatio...». La pittura infamante nei secoli XIII-XVI, Roma, Jouvence, I 979 . 
90. La sfortuna del Capitolo della scomunica trova conferma in un elenco di Componimenti poetici in lode o in biasimo d'alcuna cosa redatto da Antonio Maria Biscioni dopo il 22 giugno r 669 e finito tra le Giunte alla Toscana letterata del Cinelli nel cod. Magl. IX 82, pp. I 83-84. Si tratta di poco più di un appunto sommario: tra le omissioni e gli errori uno riguarda Molza, registrato come autore solo dei capitoli Della insalata e Dei fichi.

9г. La riproduzione del codice è disponibile sul sito della Philadelphia University http://dla.library.upenn.edu. Oltre ai capitoli contiene, non indicati nella tavola posta in principio, Chiome d'argento fino, irte ed attorte e O spirito bizzarro del Pistoia di Berni e Se dio vi guardi e vi mantenga sano di Antonfrancesco Grazzini ad Agnolo Firenzuola, con il responsivo Non è però quest'abito sì strano.

92. Non considero qui né altrove il Magl. VII i I 96, contenente la trascrizione del Libro primo delle Opere burlesche Firenze, Giunti, I 548, autografo di Lionardo Salviati con inserti altrui.

93. E. Garavelui, I pentimenti di Ser Agresto. Terza variazione sul Commento alla Ficheide di Annibal Caro.

94. La tavola in I mss. Landau Finaly della Biblioteca Nazionale Centrale di Firenze. Catalogo, a cura di G. Lazzi - M. Rolih Scarlino, I, Firenze-Milano, Giunta Regionale Toscana - Editrice Bibliografica, I994, pp. 243-52.

95. Sulla tradizione a stampa delle rime burlesche nel XVI-XVII secolo cfr. D. Romei, Ricezione della poesia del Cinquecento: la «fortuna editoriale», in Il poeta e il suo pubblico. Lettura e commento dei testi lirici nel Cinquecento. Convegno internazionale di studi (Ginevra, I5-I7 maggio 2008), a cura di M. Danzi - R. Leporatti, Genève, Droz, 20I 2, pp. 273-9I.

96. L'incipit del verso ha l'aria di una geminazione del v. I78 «Forsi che costa molto o che bisogna». Poiché entrambe le redazioni della frase non danno senso nel contesto, è probabile che $\gamma$ intervenga maldestramente per sanare un guasto dell'archetipo. "Bere" significa, in codice, avere un rapporto sessuale, e "dare buon bere" è locuzione comune per "fornire un rapporto sessuale appagante" (cfr. A. Firenzuola, Capitolo in lode della sete; A. Grazzini, Capitolo in lode della salsiccia, v. I 54 «e reca dopo sé migliore il bere»). A questo punto del capitolo di Molza non è pensabile la constatazione che l'insalata "non dia buon bere", così come neppure viene a taglio l'interrogativo retorico "se dia buon bere". Ai vv. I 86-89 si legge infatti che, sebbene penda il giudizio se sia migliore l'insalata o il melone (il coito de retro), gli intenditori non esitano a preferire la prima, affermazione possibile solo dopo che ne sia stata dichiarata senza riserve la bontà.

97. Ludi esegetici (Berni, Comento alla Primiera - Lasca, Piangirida e Comento di maestro Niccodemo sopra il Capitolo della salsiccia), a cura di M. Plaisance - D. RomeiF. Pignatti, Manziana, Vecchiarelli, 2005, p. 299.

98. VARCHI, Hercolano, p. 854. 